\title{
Semi-Analytical Methods for Different Problems of Diffraction-Radiation by Vertical Circular Cylinders
}

\author{
Šime Malenica $^{1 *}$ \\ ${ }^{\text {I}}$ Sime Malenica, Bureau Veritas, Research Department, Paris, France
}

(Manuscript Received March 5 2012; Revised April 16, 2012; Accepted May 25, 2012)

\begin{abstract}
As in the other fields of mechanics, analytical methods represent an important analysis tool in marine hydrodynamics. The analytical approach is interesting for different reasons : it gives reference results for numerical codes verification, it gives physical insight into some complicated problems, it can be used as a simplified predesign tool, etc. This approach is of course limited to some simplified geometries (cylinders, spheres, ...), and only the case of one or more cylinders, truncated or not, will be considered here. Presented methods are basically eigenfunction expansions whose complexity depends on the boundary conditions. The hydrodynamic boundary value problem (BVP) is formulated within the usual assumptions of potential flow and is additionally simplified by the perturbation method. By using this approach, the highly nonlinear problem decomposes into its linear part and the higher order (second, third, ...) corrections. Also, periodicity is assumed so that the time dependence can be factorized i.e. the frequency domain formulation is adopted. As far as free surface flows are concerned, only cases without or with small forward speed are sufficiently simple to be solved semi-analytically. The problem of the floating body advancing in waves with arbitrary forward speed is far more complicated. These remarks are also valid for the general numerical methods where the case of arbitrary forward speed, even linearized, is still too difficult from numerical point of view, and "it is fair to say that there exists at present no general practical numerical method for the wave resistance problem" [9], and even less for the general seakeeping problem. We note also that, in the case of bluff bodies like cylinders, the assumptions of the potential flow are justified only if the forward speed is less than the product of wave amplitude with wave frequency.
\end{abstract}

Keywords: Semi-analytical method, Third order diffaction, Hydroelasticity, Bottom mounted cylinder, Eigen-function expansion

\section{Introduction}

As in the other fields of mechanics, analytical methods represent an important analysis tool in marine hydrodynamics. The analytical approach is interesting for different reasons : it gives reference results for numerical codes verification, it gives physical insight into some complicated problems, it can be used as a simplified predesign tool, etc. This approach is of course limited to some simplified geometries (cylinders, spheres, ...), and only the case of one or

\footnotetext{
*Corresponding author. Tel.: +33-6-71-91-31-39, Fax.: +33-1-55-24-70-26,

E-mail address: sime.malenica@bureauveritas.com

Copyright $\odot$ KSOE 2012.
}

more cylinders, truncated or not, will be considered here. Presented methods are basically eigenfunction expansions whose complexity depends on the boundary conditions. The hydrodynamic boundary value problem (BVP) is formulated within the usual assumptions of potential flow and is additionally simplified by the perturbation method. By using this approach, the highly nonlinear problem decomposes into its linear part and the higher order (second, third, ...) corrections. Also, periodicity is assumed so that the time dependence can be factorized i.e. the frequency domain formulation is adopted. As far as free surface flows are concerned, only cases without or with small forward speed are sufficiently simple to 
be solved semi-analytically. The problem of the floating body advancing in waves with arbitrary forward speed is far more complicated. These remarks are also valid for the general numerical methods where the case of arbitrary forward speed, even linearized, is still too difficult from numerical point of view, and "it is fair to say that there exists at present no general practical numerical method for the wave resistance problem" [9], and even less for the general seakeeping problem. We note also that, in the case of bluff bodies like cylinders, the assumptions of the potential flow are justified only if the forward speed is less than the product of wave amplitude with wave frequency.

\section{General Theory}

Classical Assumptions of perfect fluid and irrotational flow inside the fluid domain $\Omega$, are adopted. We define the right-handed coordinate system $(x$, $y, z$ ) fixed to the body, with $z=0$ the undisturbed free surface, the axis $\mathrm{z}$ pointing upward. The sea bottom $S_{\mathrm{BT}}$ is assumed to be the horizontal plane placed at $z=$ $-H$.

With these assumptions, a nonlinear boundary value problem for the velocity potential $\phi(x, y, z)$ can be formulated :

$$
\begin{aligned}
& \Delta \Phi=0, \quad \mathrm{x} \in \Omega \\
& (\nabla \Phi-\mathrm{v}) \mathrm{n}=0, \mathrm{x} \in S_{B} \cup S_{B T} \\
& \frac{\partial^{2} \Phi}{\partial t^{2}}+g \frac{\partial \Phi}{\partial z}+2 \nabla \Phi \nabla \frac{\partial \Phi}{\partial t}+\frac{1}{2} \nabla \Phi \nabla(\nabla \Phi \nabla \Phi)=0 \\
& z=\Xi
\end{aligned}
$$

where $\Xi$ is the exact position of the free surface defined by :

$$
\Xi=-\frac{1}{g}\left(\frac{\partial \Phi}{\partial t}+\frac{1}{2} \nabla \Phi \nabla \Phi\right), \quad z=\Xi
$$

and $\mathbf{v}$ is the velocity of the rigid boundaries.

The above BVP should be completed by and appropriate radiation condition at infinity. The convention used throughout this paper is that the normal vector $\mathbf{n}$ is pointing out of the fluid domain.

In order to simplify the BVP (especially the free surface condition) we proceed in two steps. First we assume the displacements to be small and we express the quantities at the instantaneous position by Taylor series developments with respect to their mean position, and after that we introduce the perturbation se- ries with respect to the wave steepness $\left(\varepsilon=k_{0} A\right.$ with $k_{0}$ - wave number, $A$ - wave amplitude ) which allows the decomposition of the nonlinear problem into the less complicated first, second, third, ... order approximations.

Even if the methodology remains the same for $U=0$ and $U \neq 0$ there are some differences in the application, so we consider both cases separately.

\subsection{The Zero Forward Speed Case}

In the case of no forward speed, the Taylor series expansion gives for the free surface elevation :

$\Xi=-\frac{1}{g}\left(\frac{\partial \Phi}{\partial t}+\frac{1}{2} \nabla \Phi \nabla \Phi-\frac{1}{g} \frac{\partial \Phi}{\partial t} \frac{\partial^{2} \Phi}{\partial t \partial z}\right)+O\left(\Phi^{3}\right)$

and for the free surface condition :

$$
\begin{aligned}
& \frac{\partial^{2} \Phi}{\partial t^{2}}+g \frac{\partial \Phi}{\partial z}= \\
& -2 \nabla \Phi \nabla \frac{\partial \Phi}{\partial t}-\frac{1}{2} \nabla \Phi \nabla(\nabla \Phi \nabla \Phi) \\
& +\frac{1}{g} \frac{\partial \Phi}{\partial t}\left(\frac{\partial^{3} \Phi}{\partial t^{2} \partial z}+g \frac{\partial^{2} \Phi}{\partial z^{2}}\right) \\
& +\frac{2}{g} \frac{\partial \Phi}{\partial t}\left(\nabla \frac{\partial \Phi}{\partial z} \nabla \frac{\partial \Phi}{\partial t}+\nabla \Phi \nabla \frac{\partial^{2} \Phi}{\partial t \partial z}\right) \\
& -\frac{1}{g}\left(\frac{1}{g} \frac{\partial \Phi}{\partial t} \frac{\partial^{2} \Phi}{\partial t \partial z}-\frac{1}{2} \nabla \Phi \nabla \Phi\right)\left(\frac{\partial^{3} \Phi}{\partial t^{2} \partial z}+g \frac{\partial^{2} \Phi}{\partial z^{2}}\right) \\
& -\frac{1}{2 g^{2}}\left(\frac{\partial \Phi}{\partial t}\right)^{2}\left(\frac{\partial^{4} \Phi}{\partial t^{2} \partial z^{2}}+g \frac{\partial^{3} \Phi}{\partial z^{3}}\right)+O\left(\Phi^{4}\right)
\end{aligned}
$$

In this case the perturbation series for the potential has the following form :

$$
\Phi=\varepsilon \emptyset^{(1)}+\varepsilon^{2} \emptyset^{(2)}+\varepsilon^{3} \emptyset^{(3)}+O\left(\varepsilon^{4}\right)
$$

Also we assume time periodicity at frequency $\omega$ for the flow at first order :

$$
\varepsilon \emptyset^{(1)}=\mathfrak{R}\left\{\varphi^{(1)} e^{-i \omega t}\right\}
$$

From which we easily deduce the form of the higher order potentials :

$$
\begin{aligned}
& \varepsilon^{2} \emptyset^{(2)}=\bar{\varphi}^{(2)}+\mathfrak{R}\left\{\varphi^{(2)} e^{-2 i \omega t}\right\} \\
& \varepsilon^{3} \emptyset^{(3)}=\mathfrak{R}\left\{\bar{\varphi}^{(3)} e^{-i \omega t}\right\}+\mathfrak{R}\left\{\varphi^{(3)} e^{-3 i \omega t}\right\}
\end{aligned}
$$

The similar perturbation series is assumed for the free surface elevation $\Xi$ (and all other quantities of interest) : 


$$
\begin{aligned}
\Xi= & \varepsilon \Xi^{(1)}+\varepsilon^{2} \Xi^{(2)}+\varepsilon^{3} \Xi^{(3)}+O\left(\varepsilon^{4}\right) \\
= & \Re\left\{\eta^{(1)} e^{-i \omega t}\right\}+\bar{\eta}^{(2)}+\mathfrak{R}\left\{\bar{\eta}^{(2)} e^{-2 i \omega t}\right\} \\
& +\mathfrak{R}\left\{\bar{\eta}^{(3)} e^{-i \omega t}\right\}+\mathfrak{R}\left\{\eta^{(3)} e^{-3 i \omega t}\right\}+O\left(\varepsilon^{4}\right)
\end{aligned}
$$

After introduction of the perturbation series $(7,11)$ in (6) and (5) we obtain the following free surface conditions and free surface elevations at the corresponding orders :

$O(\varepsilon)$

$-v \varphi^{(1)}+\frac{\partial \varphi^{(1)}}{\partial z}=0$

$\eta^{(1)}=\frac{i \omega}{g} \varphi^{(1)}$

$O\left(\varepsilon^{2}\right)$

$$
\begin{aligned}
-4 v \varphi^{(2)}+ & \frac{\partial \varphi^{(2)}}{\partial z}=\frac{i \omega}{g}\left[\nabla \varphi^{(1)} \nabla \varphi^{(1)}\right. \\
& \left.-\frac{1}{2} \varphi^{(1)}\left(\frac{\partial^{2} \varphi^{(1)}}{\partial z^{2}}-v \frac{\partial \varphi^{(1)}}{\partial z}\right)\right]
\end{aligned}
$$

$\eta^{(2)}=\frac{2 i \omega}{g} \varphi^{(2)}-\frac{1}{4 g} \nabla \varphi^{(1)} \nabla \varphi^{(1)}-\frac{v^{2}}{2 g} \varphi^{(1)} \varphi^{(1)}$

$O\left(\varepsilon^{3}\right)$

$$
\begin{aligned}
-9 v \phi^{(3)}+\frac{\partial \phi^{(3)}}{\partial z}= & \frac{3 i \omega}{g} \nabla \phi^{(2)} \nabla \phi^{(1)} \\
& -\frac{i \omega}{2 g}\left[\phi^{(1)}\left(\frac{\partial^{2} \phi^{(2)}}{\partial z^{2}}-4 v \frac{\partial \phi^{(2)}}{\partial z}\right)\right. \\
& \left.+2 \phi^{(2)}\left(\frac{\partial^{2} \phi^{(1)}}{\partial z^{2}}-v \frac{\partial \phi^{(1)}}{\partial z}\right)\right] \\
& -\frac{1}{8 g} \nabla \phi^{(1)} \nabla\left(\nabla \phi^{(1)} \nabla \phi^{(1)}\right) \\
& -\frac{v}{g} \phi^{(1)} \nabla \phi^{(1)} \nabla \frac{\partial \phi^{(1)}}{\partial z} \\
& +\frac{1}{4 g}\left(v \phi^{(1)} \frac{\partial \phi^{(1)}}{\partial z}+\frac{1}{2} \nabla \phi^{(1)} \nabla \phi^{(1)}\right) \\
& \left(\frac{\partial^{2} \phi^{(1)}}{\partial z^{2}}-v \frac{\partial \phi^{(1)}}{\partial z}\right)
\end{aligned}
$$

where $v=\omega^{2} / g$.

Of course, all these potentials must satisfy the Laplace equation in the fluid domain, zero normal velocity on the fixed boundaries and corresponding radiation conditions which will be discussed later.

We precise that only the first order quantities at frequency $\omega$, the second order quantities at frequency $2 \omega$ and the third order quantities at frequency $3 \omega$ will be considered here.

\subsubsection{Wave Loads}

They are obtained by integrating pressure over the wetted surface of the body :

$$
\mathbf{F}=\int_{\mathrm{S}_{\mathrm{B}}} p \mathbf{n} d S
$$

where the pressure is calculated from the Bernoulli equation :

$$
p=-\varrho g z-\varrho \frac{\partial \Phi}{\partial t}-\frac{1}{2} \varrho(\nabla \Phi)^{2}
$$

Careful analysis should be performed to collect the terms of different order. The problem is simplified for fixed bodies and following expressions can be obtained :

$O(\varepsilon)$

$$
\mathbf{F}^{(1)}=\int_{S_{B 0}} i \omega \varrho \varphi^{(1)} \mathbf{n} d S
$$

$O\left(\varepsilon^{2}\right)$

$$
\begin{aligned}
& \mathbf{F}^{(2)} \\
& =\int_{S_{B 0}}\left(2 i \omega \varrho \varphi^{(2)}\right. \\
& \left.-\frac{1}{4} \varrho \nabla \varphi^{(1)} \nabla \varphi^{(1)}\right) \mathrm{n} d S \\
& +\frac{1}{4} \varrho g \int_{C_{B 0}} \eta^{(1)} \eta^{(1)} \mathrm{n} d C
\end{aligned}
$$


$O\left(\varepsilon^{3}\right)$

$$
\boldsymbol{F}^{(3)}=\int_{\mathrm{S}_{B 0}}\left(3 i \omega \varrho \varphi^{(3)}\right.
$$

$$
\begin{aligned}
& \left.-\frac{1}{2} \varrho \nabla \varphi^{(1)} \nabla \varphi^{(2)}\right) \mathrm{n} d S \\
& +\frac{1}{2} \varrho g \int_{C_{B 0}} \eta^{(1)}\left(\eta^{(2)}\right. \\
& \left.-\frac{v}{4} \eta^{(1)} \eta^{(1)}\right) \mathrm{n} d C
\end{aligned}
$$

\subsection{The Small Forward Speed Case}

In this case only the linear problem will be considered. Due to the presence of small forward speed $U$ in the positive $x$ direction (or the current in the negative $x$ direction), the perturbation series has the following form :

$$
\begin{aligned}
& \Phi=U \bar{\phi}^{(0)}+\varepsilon \phi^{u}+O\left(\varepsilon^{2}\right), \\
& \Xi=U \bar{\Xi}^{(0)}+\varepsilon \Xi^{u}+O\left(\varepsilon^{2}\right)
\end{aligned}
$$

where $\bar{\emptyset}^{(0)}$ is the steady potential due to the presence of current, and can be further decomposed to the uniform current and the body perturbation $\bar{\phi}, \bar{\phi}^{(0)}=\bar{\phi}-x$. Since we have chosen to describe the problem in the coordinate system $(x, y, z)$ fixed to the body the incident potential can be written as :

$$
\begin{aligned}
& \varepsilon \varphi_{I}^{(1)}(x, t) \\
& =\mathfrak{R}\left\{-\frac{i g A}{\omega} \frac{\cosh k_{0}(z+H)}{\cosh k_{0} H} e^{i k_{0}(x \cos \beta+y \sin \beta)} e^{-i\left(\omega-k_{0} U \cos \beta\right) t}\right\} \\
& =\mathfrak{R}\left\{\phi_{I}^{(1)} e^{-i \omega_{e} t}\right\}
\end{aligned}
$$

where $\omega_{e}$ denotes the well known encounter frequency $\left(\omega_{e}=\omega-k_{o} U \cos \beta\right), \beta$ is the incidence angle i.e. angle between the direction of wave propagation and the positive $x$ axis. $A$ is the amplitude of the wave, $\omega$ is its frequency in the earth fixed coordinate system and $k_{o}$ is its wavenumber $v=k_{o} \tanh k_{o} H$. This allows us to write the following expressions for the potential and free surface elevation :

$$
\begin{aligned}
& \propto \phi^{u}(x, t)=\mathfrak{R}\left\{\varphi^{u}(x) e^{-i \omega_{e} t}\right\} \\
& \varepsilon \Xi^{u}=\mathfrak{R}\left\{\eta^{u} e^{-i \omega_{e} t}\right\}
\end{aligned}
$$

After perturbing the original BVP (3) we obtain at different orders :

$O(1)$

$$
\begin{aligned}
& \frac{\partial \varphi^{(0)}}{\partial z}=0 \\
& \bar{\Xi}^{(0)}=0
\end{aligned}
$$

$O(\varepsilon)$

$-\omega_{e}^{2} \phi^{u}-2 i \omega_{e} U \nabla_{0} \bar{\phi}^{(0)} \nabla_{0} \varphi^{u}+g \frac{\partial \phi^{u}}{\partial_{z}}+i \omega_{e} U \phi^{u} \frac{\partial^{2} \bar{\phi}^{(0)}}{\partial z^{2}}=0$

$$
\eta^{u}=-\frac{1}{g}\left[-i \omega_{e} \varphi^{u}+U \nabla_{0} \phi^{(0)} \nabla_{0} \varphi^{u}\right]
$$

where $\nabla_{0}$ denotes horizontal gradient $\nabla_{0}=$ $\left(\frac{\partial}{\partial x}, \frac{\partial}{\partial y}, 0\right)$.

In this section we will also consider the case of a freely floating body so that the radiation problem must also be considered. We write first the general decomposition of the total potential $\varphi^{u}$ into the incident $\varphi_{I}^{u}$, diffracted $\varphi_{D}^{u}$ and the radiated $\varphi_{R j}^{u}$ parts :

$\varphi^{u}=\varphi_{I}^{(1)}+\varphi_{D}^{u}-i \omega_{e} \sum_{j=1}^{6} \xi_{j}^{u} \varphi_{R j}^{u}$

where $\xi_{j}^{u}$ stands for the body displacements.

According to this decomposition, the following body boundary conditions for different potentials can be deduced :

$$
\frac{\partial \varphi_{D}^{u}}{\partial n}=-\frac{\partial \varphi_{I}^{(1)}}{\partial n}, \frac{\partial \varphi_{R j}^{u}}{\partial n}=n_{j}+\frac{i U}{\omega_{e}} m_{j}
$$

where :

$\left(n_{1,}, n_{2} ; n_{3}\right)=\mathrm{n},\left(n_{4}, n_{5}, n_{6}\right)=x \wedge \mathrm{n}$

$\left(m_{1}, m_{2}, m_{3}\right)=-(\mathrm{n} \cdot \nabla) \nabla \bar{\phi}^{(0)}$

$\left(m_{4}, m_{5}, m_{6}\right)=-(\mathrm{n} \cdot \nabla)\left(x \wedge \nabla \bar{\phi}^{(0)}\right)$ 
On the free surface we have :

$$
\begin{aligned}
& -\omega_{e}^{2} \phi_{R j}^{u}-2 i \omega_{e} U \nabla_{0} \bar{\phi}^{(0)} \nabla_{0} \phi_{R j}^{u}+g \frac{\partial \phi_{R j}^{u}}{\partial z} \\
& +i \omega_{e} U \phi_{R j}^{u} \frac{\partial^{2} \bar{\phi}^{(0)}}{\partial z^{2}}=0
\end{aligned}
$$

$$
-\omega_{e}^{2} \phi_{D}^{u}+g \frac{\partial \phi_{D}^{u}}{\partial z}+2 i \omega_{e} U \frac{\partial \phi_{D}^{u}}{\partial x}
$$

$-2 i \omega_{e} U \nabla_{0} \bar{\phi} \nabla_{0}\left(\phi_{D}^{u}+\phi_{1}^{(1)}\right)+i \omega_{e} U\left(\phi_{D}^{u}+\phi_{1}^{(1)}\right) \frac{\partial^{2} \bar{\phi}}{\partial z^{2}}=0$

The small forward speed problem defined above can be solved directly in that form but usually new perturbation series with respect to forward speed parameter $\tau=U \omega / g$ is introduced (the incident potential being not affected by this perturbation) :

$$
\varphi^{u}=\varphi^{(1)}+\tau \varphi^{(11)}+O\left(\tau^{2}\right)
$$

The free surface condition decomposes into :

$$
-v \varphi^{(1)}+\frac{\partial \varphi^{(1)}}{\partial z}=0
$$

$O(\tau)$

$$
\begin{aligned}
& -v \phi^{(11)}+\frac{\partial \phi^{(11)}}{\partial z}=-2 i \frac{\partial \phi^{(1)}}{\partial x}-2 k_{0} \cos \beta \phi^{(1)} \\
& +2 i \nabla_{0} \bar{\phi} \nabla_{0} \phi^{(1)}-i \phi^{(1)} \frac{\partial^{2} \bar{\phi}}{\partial z^{2}}
\end{aligned}
$$

and the free surface elevation $\eta^{u}=\eta^{(1)}+\tau \eta^{(11)}$ to :

$$
\begin{aligned}
\eta^{(1)} & =\frac{i \omega}{g} \varphi^{(1)} \\
\eta^{(1)} & =\frac{i \omega}{g}\left[\phi^{(11)}-\frac{k_{0}}{v} \cos \beta \phi^{(1)}+\frac{i}{v} \nabla_{0} \bar{\phi}^{(0)} \nabla_{0} \phi^{(1)}\right]
\end{aligned}
$$

The inconvenience of this approach is that the additional perturbation series (35) causes the secularity of the solution, i.e. the unphysical growth for $R \rightarrow \infty$. It has been shown in [18] that this problem can be solved using the multiple scale analysis.

We note here that the potential $\varphi^{(1)}$ is exactly the linear potential from the zero forward speed case, and the potential $\varphi^{(11)}$ is its first order correction with respect to the forward speed. Further onward, this potential will be decomposed into two parts. In fact, an explicit particular solution satisfying the free surface condition :

$$
-v \phi_{p}^{(11)}+\frac{\partial \phi_{p}^{(11)}}{\partial z}=-2 i \frac{\partial \phi^{(11)}}{\partial x}-2 k_{0} \cos \beta \phi^{(1)}
$$

and Laplace equation in the fluid, can be found [8. 16]. One of the possible expressions is :

$\varphi_{p}^{(11)}=-2\left(i \frac{\partial}{\partial x}+k_{0} \cos \beta\right) \frac{\partial \varphi^{(1)}}{\partial v}$

A homogeneous solution should be added to the particular solution above, in order to annul the normal velocity on the cylinder, induced by $\varphi_{p}^{(11)}$. This homogeneous part of the solution is of the same type as $\varphi^{(1)}$ and the same method can be used for its evaluation. The remaining part of the free surface condition (37) will be treated in the same way as $\varphi_{D}^{(2)}$ and $\varphi_{D}^{(3)}$ because no particular solution equivalent to (41) can be found. However, due to the rapid decay of the forcing function $\left(Q=2 i \nabla_{0} \bar{\phi} \nabla_{0} \varphi^{(1)}-i \varphi^{(1)} \frac{\partial^{2} \bar{\phi}}{\partial z^{2}}\right)$ the radiation condition for this part (denoted $\varphi_{q}^{(11)}$ ) of the potential $\varphi^{(11)}$ will be of the Sommerfield type (69).

\subsubsection{Wave Loads}

In the case of small forward speed the situation is complicated by the presence of the steady potential but is simplified because we limited ourselves to the linear and steady second order loads, both of which can be obtained by knowing only the first order potential $\varphi^{\mathrm{u}}$ and the steady potential $\bar{\phi}$.

\section{First order loads}

The following expression for the first order force can be obtained :

$$
\mathrm{F}^{u}=-\tilde{n} \iint_{S_{B 0}}\left(-i \omega_{e} \phi^{u}+U \nabla \bar{\varphi}^{(0)} \nabla \phi^{u}\right) \mathrm{n} d S
$$

The global forces are usually decomposed into the so called excitation force associated with the diffracted and incident potential, and the radiation force which is written in the form of 
added mass and damping coefficients :

$$
\begin{aligned}
& F_{i}^{(1)}=-\tilde{n} \iint_{S_{B o}}\left[-i \omega_{e}\left(\phi_{I}^{(1)}+\phi_{D}^{u}\right)\right. \\
& \left.+U \nabla \bar{\varphi}^{(0)} \nabla\left(\phi_{I}^{(1)}+\phi_{D}^{u}\right)\right] n_{i} d S
\end{aligned}
$$

$$
\omega_{e}^{2} a_{i j}^{u}+i \omega_{e} b_{i j}^{u}=\tilde{n} \iint_{S_{B o}}\left(\omega_{e}^{2} \phi_{R j}^{u}+i \omega_{e} U \nabla \bar{\varphi}^{(0)} \nabla \phi_{R j}^{u}\right) n_{i} d S
$$

\section{- $\quad$ Steady second order loads}

The steady second order loads are important for the analysis of the slow drift motions of moored floating structures. The case of small forward speed is particularly important because it permits the evaluation of one component of the slow drift damping, namely the wave drift damping. In fact this damping is defined as the derivative of the steady second order force with respect to the forward speed, and it can be calculated only after the resolution of the small forward speed diffraction-radiation problem. Only horizontal components of the steady second order forces will be considered here and the so called far-field expression for these forces is [26]:

$$
\begin{aligned}
& \overline{\mathbf{F}}^{u}=\frac{\varrho}{4} \Re\left\{\iint_{S_{B o}}\left[\nabla \varphi^{u} \nabla \varphi^{u *} \mathbf{n}_{0}-2 \nabla_{0} \varphi^{u} \frac{\partial \varphi^{u *}}{\partial n}\right] d S\right. \\
& \left.-\frac{\omega_{e}^{2}}{g} \int_{C_{B o}} \varphi^{u} \varphi^{u *} \mathbf{n}_{0} d C\right\}
\end{aligned}
$$

The wave drift damping coefficient $\mathbf{B}=-\partial \overline{\mathbf{F}}^{u} / \partial U$ is usually obtained by numerical differentiation after calculating $\overline{\mathbf{F}}^{\mathbf{u}}$ for two small forward speed. Compared to the case without forward speed the evaluation of the steady second order forces is much more complicated and that is why some authors tried to propose some simplified methods to quantify these loads. An extremely simple 3D formula for wave drift damping coefficient was proposed in [7]. It turned out that, in the case of the fixed single or group of bottom mounted cylinders, this formula gives exactly the same results as the complicated theory presented here. Theoretical proof of the formula was proposed in a controversial paper [2] but it is not clear yet if the formula effectively should work for the general case, and one of the conclusions of the present paper is that it does not. Anyway, we recall here the expression for the $x$ component of the wave drift damping coefficient $B_{11}$ for finite water depth [16] :

$$
\begin{aligned}
& B_{11}=\left\{\left[\left(\frac{\partial \bar{F}_{1}(\omega)}{\partial \omega}-\frac{1}{\alpha} \frac{\partial \alpha}{\partial \omega} \bar{F}_{1}(\omega)\right) \omega+\frac{2}{\alpha} \bar{F}_{1}(\omega)\right]\right. \\
& \left.\cos \beta-\frac{1}{\alpha} \frac{\partial \bar{F}_{1}(\omega)}{\partial \beta} \sin \beta\right\} \frac{k_{0}}{\omega}
\end{aligned}
$$

where $\bar{F}_{1}$ is the zero forward speed steady second order force (so called drift force), $\beta$ is the wave incidence and $\alpha$ is the ratio between the group velocity and phase velocity of waves $\alpha=1 / 2+k_{0} H /$ $\sinh 2 k_{0} H$. The utility of the formula is evident because only the zero forward speed case needs to be solved which becomes nowadays quite a trivial task.

\subsection{Hydroelasticity}

Hydroelasticity contains another type of problems where the presented methods can be used. It concerns the bending of a vertical column under the action of waves. In fact, hydroelasticity is an important problem for large floating structures which have their natural frequencies sufficiently small to be excited by the common wave spectra. The elastic displacements of the body become of the same order as the rigid body displacements and should be treated together. The method we use to treat this kind of problem is explained in more details in [20] and consists in coupling the beam finite element structural model with the $3 \mathrm{D}$ hydrodynamic model. Here we recall just the basic steps.

\subsubsection{Theoretical Assumption}

The cylinder is assumed to be a slender beam with the horizontal displacement $W(z, t)=\Re\left\{w(z) e^{-i \omega t}\right\}$ along the height. Differential equation for the structural deflection of the beam, in the simpliest case, can be written in the form [27] :

$-\omega^{2} m w+\frac{d^{2}}{d z^{2}}\left(E I \frac{d^{2} w}{d z^{2}}\right)=q$

where $m(z)$ is the mass distribution, $E(z)$ is the modulus of elasticity, $I(z)$ is cross-sectional moment of inertia and $q(z)$ is the local pressure force acting on a horizontal section of the cylinder.

The numerical method used here is the well known finite element method based on the so called "displacement" formulation. 


\subsubsection{Beam Finite Element}

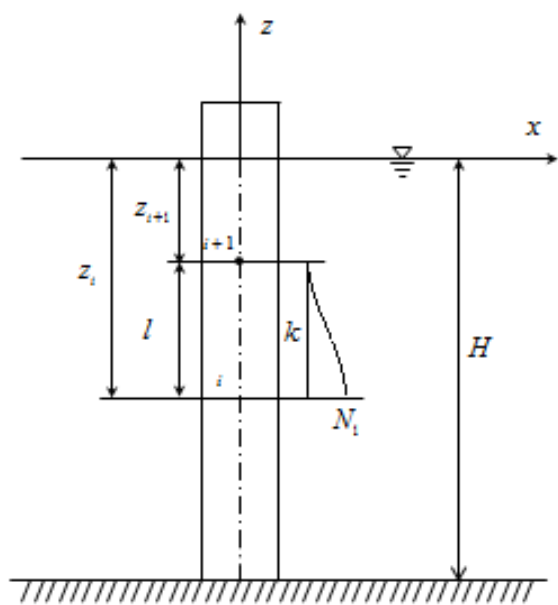

Fig. 1. Finite element model

As far as the structural problem is concerned, the theory we use is the classical one [27] and is repeated here only for completeness. We define an isolated finite element $k$ of length $l$ and we assume the displacement $w^{k}(z)$ (in the local coordinate system) within the element in the following form :

$w^{k}(z)=\sum_{i=1}^{4} N_{i}^{k}(z) \delta_{i}^{k}=\{N\}^{k T}\{\delta\}^{k}$

where ${ }^{T}$ signify the transpose operation, $\{\delta\}^{k}$ is the vector of nodal displacements $w_{i}^{k}$ and nodal slopes $\theta_{i}^{k}=(\partial w / \partial z)_{i}^{k}$, and $\{N\}^{k}$ is the vector of the shape functions :

$\{\delta\}^{k}=\left\{\begin{array}{l}w_{1}^{k} \\ \theta_{1}^{k} \\ w_{2}^{k} \\ \theta_{2}^{k}\end{array}\right\},\{N\}^{k}=\left\{\begin{array}{l}1-3\left(\frac{z}{l}\right)^{2}+2\left(\frac{z}{l}\right)^{3} \\ l\left[\left(\frac{z}{l}\right)-2\left(\frac{z}{l}\right)^{2}+\left(\frac{z}{l}\right)^{3}\right] \\ 3\left(\frac{z}{l}\right)^{2}-2\left(\frac{z}{l}\right)^{3} \\ l\left[-\left(\frac{z}{l}\right)^{2}+\left(\frac{z}{l}\right)^{3}\right]\end{array}\right\}$

After the discretisation of the equation (47) using the method of the beam potential energy minimization, we obtain the well known matrix formulation of the problem :

$$
\left(-\omega^{2}[M]^{k}+[K]^{k}\right)\{\delta\}^{k}=\{f\}^{k}+\{F\}^{k}
$$

The matrix $[M]^{k}$ and $[K]^{k}$ denote respectively mass and stiffiness matrix of the beam, with the following generic elements :

$$
m_{i j}^{k}=m^{k} \int_{0}^{l} N_{i}^{k} N_{j}^{k} d z, k_{i j}^{k}=E^{k} I^{k} \int_{0}^{l} \frac{d^{2} N_{i}^{k}}{d z^{2}} \frac{d_{2} N_{j}^{k}}{d z^{2}} d z
$$

within $E^{k} I^{k}$ denoting the averaged value of the stiffness factor of the element and $m^{k}$ the averaged mass per unit length.

After performing the integration in (51) the following expressions are obtained :

$$
\begin{gathered}
{[K]^{k}=\frac{2 E^{k} I^{k}}{l^{3}}\left[\begin{array}{cccc}
6 & 3 l & -6 & 3 l \\
3 l & 2 l^{2} & -3 l & l^{2} \\
-6 & -3 l & 6 & -3 l \\
3 l & l^{2} & -3 l & 2 l^{2}
\end{array}\right],} \\
{[M]^{k}=\frac{m^{k} l}{420}\left[\begin{array}{cccc}
156 & 22 l & 54 & -13 l \\
22 l & 4 l^{2} & 13 l & -3 l^{2} \\
54 & 13 l & 156 & -22 l \\
-13 l & -3 l^{2} & -22 l & 4 l^{2}
\end{array}\right]}
\end{gathered}
$$

The vector $\{F\}^{k}$ is the vector of the concentrated forces and moments at the ends of the element :

$\{F\}^{k}=\left\{\begin{array}{c}Q_{1}^{k} \\ M_{1}^{k} \\ Q_{2}^{k} \\ M_{2}^{k}\end{array}\right\}$

Finally the vector $\{f\}^{k}$ represents the action of the distributed external forces which are in our case due to the water pressure. The elements $f_{i}^{k}$ of this vector are obtained by the following expression :

$f_{i}^{k}=\int_{0}^{l} q^{k} N_{i}^{k} d z$

As it will be shown later, the external forces distribution $q^{k}(\mathrm{z})$ is obtained by solving the hydrodynamic problem.

\subsubsection{Assembly}

Once the characteristic matrix and vectors of each element is calculated, the assembling is performed and the following linear system of equations is obtained :

$\left(-\omega^{2}[\mathrm{M}]+[\mathrm{K}]\right)\{\delta\}=\{\mathrm{f}\}+\{\mathrm{F}\}$

The assembly procedure is the classical one [27] and will not be repeated here. We note just that the dimension of the linear system of equations is $2\left(N_{e}+1\right)$ 
and that the global displacement vector is :

$$
\begin{aligned}
\{\delta\} & =\left\{\delta_{1}, \delta_{2}, \cdots, \delta_{i}, \delta_{i+1}, \cdots, \delta_{2 N_{e}+1}, \delta_{2 N_{e}+2}\right\}^{T} \\
& =\left\{w_{1}, \theta_{1}, w_{2}, \theta_{2}, \cdots, w_{i}, \theta_{i}, w_{i+1}, \theta_{i+1}, \cdots, w_{N_{e}}, \theta_{N_{e}}\right. \\
& \left., w_{N_{e+1}}, \theta_{N_{e}+1}\right\}^{T}
\end{aligned}
$$

In the case of "dry" structures the resulting matrix $[\mathbf{M}]$ and $[\mathbf{K}]$ are banded around the diagonal with the half-band width equal to 4 . The vector $\{\mathbf{F}\}$ is the vector of the concentrated external loads, if any.

\subsubsection{Hydrodynamic problem}

The hydrodynamic problem is considered within the usual assumptions of the potential flow and the linear case is considered only. The total potential $\varphi^{(1)}$ is decomposed into incident $\varphi_{I}^{(1)}$, diffracted $\varphi_{D}^{(1)}$ part, and a part due to the cylinder displacements $\varphi_{R}^{(1)}$. We write :

$$
\begin{aligned}
\varphi^{(1)} & =\varphi_{I}^{(1)}+\varphi_{D}^{(1)}-i \omega w \varphi_{R}^{(1)} \\
& =\varphi_{I}^{(1)}+\varphi_{D}^{(1)}-i \omega \sum_{k=1}^{N_{c}} \sum_{j=1}^{4} \delta_{j}^{k} \varphi_{R j}^{(1) k}
\end{aligned}
$$

The boundary condition on the body surface $\partial \varphi^{(1)} / \partial n=-i \omega w(z) n_{x}$ gives the following conditions for different potentials :

$\frac{\partial \varphi_{D}^{(1)}}{\partial n}=-\frac{\partial \varphi_{I}^{(1)}}{\partial n}, \frac{\partial \varphi_{R j}^{(1) k}}{\partial n}=-N_{j}^{k}(z) \cos \theta$

The BVP's are completed by the Laplace equation in the fluid domain, radiation condition for diffracted and radiated parts and the following free surface condition :

$-\omega^{2} \varphi^{(1)}+g \frac{\partial \varphi^{(1)}}{\partial z}=0$

We note that the diffraction potential is exactly the first order diffraction potential without forward speed and that the radiation potentials satisfy similar kind of the BVP as those for the rigid body radiation, so that the same method can be used for their resolution.

The pressure is obtained from the Bernoulli's equation :

$$
p=-\varrho g z+i \omega \varrho \varphi^{(1)}
$$

and is integrated over the body surface to give the forces which compose the right hand side vector $\{\mathbf{f}\}$.
First we consider the hydrostatic part - @gz. It can be shown that, in the case of the vertical cylinder, this component can be written in the following form :

$$
f_{i}^{H k}=-\varrho g \iint_{S^{k}} \frac{d w}{d z} N_{i}^{k} x n_{x} d S=\sum_{p=1}^{N_{e}} \sum_{j=1}^{4} \delta_{j}^{p} k_{i j}^{p k}
$$

where $k_{i j}^{p k}$ is the hydrostatic stiffness matrix coefficient defined by :

$$
k_{i j}^{p k}-\varrho g a^{2} \pi \int_{0}^{1} N_{i}^{k} \frac{d N_{j}^{p}}{d z} d z
$$

with $S^{k}$ denoting the part of the column which corresponds to the $k$-th finite element.

We consider now the dynamic part of the pressure $i \omega \varrho \varphi^{(1)}$, which is first divided into the part associated with the potentials $\varphi_{I}^{(1)}$ and $\varphi_{D}^{(1)}$ independent of the body displacements and the part associated with $\varphi_{R}^{(1)}$ dependant of the displacements. We write for $\varphi_{I}^{(1)}+\varphi_{D}^{(1)}$ :

$f_{i}^{D k}=i \omega \varrho \iint_{S^{k}}\left(\varphi_{I}^{(1)}+\varphi_{D}^{(1)}\right) N_{i}^{k} n_{x} d S$

and for $\varphi_{R}^{(1)}$ :

$$
\begin{gathered}
f_{i}^{R k}=\varrho \omega^{2} \iint_{S^{k}} w \varphi_{R}^{(1)} N_{i}^{k} n_{x} d S \\
=\varrho \omega^{2} \sum_{p=1}^{N_{e}} \sum_{j=1}^{4} \delta_{j}^{p} \iint_{S^{k}} \varphi_{R j}^{(1) p} N_{i}^{k} n_{x} d S
\end{gathered}
$$

In order to write the matrix equation in the convenient form, the above expression is rewritten as :

$f_{i}^{R k}=\sum_{p=1}^{N_{e}} \sum_{j=1}^{4}\left(\omega^{2} a_{i j}^{p k}+i \omega b_{i j}^{p k}\right) \delta_{j}^{p}$

with the added mass $a_{i j}^{p k}$ and damping $b_{i j}^{p k}$ coefficients, defined as follows :

$a_{i j}^{p k}=\mathfrak{R}\left\{\tilde{n} \iint_{S^{k}} \phi_{R j}^{(1) p} N_{i}^{k} n_{x} d S\right\}$,

$b_{i j}^{p k}=\mathfrak{I}\left\{\tilde{n} \omega \iint_{S^{k}} \phi_{R j}^{(1) p} N_{i}^{k} n_{x} d S\right\}$

We note that the added mass and damping coefficients (66) have finite value for all $p$, what means that the matrix of the resulting system of equations will be 
full contrary to the case of the "dry" problem, as mentioned before (sect . 2.3.3).

The assembly of the resulting coupled system of equations is now slightly more complicated but still straightforward so we can write :

$$
\begin{aligned}
\left(-\omega^{2}\left([\mathbf{M}]+[\mathbf{A}]-i \omega[\mathbf{B}]+[\mathbf{K}]+[\mathbf{K}]^{H}\right)\right. & \{\delta\} \\
& =\{\mathbf{f}\}^{D}+\{\mathbf{F}\}
\end{aligned}
$$

The solution of this linear system of equations gives the global vector of nodal displacements which complete the solution for the potential (57).

\section{Solution for the Potentials}

We can see that, except for the stationary potential $\bar{\phi}$, all the potentials $\varphi^{(1)}, \varphi^{(2)}, \varphi^{(3)}, \varphi_{q}^{(11)}, \varphi_{R j}^{(1) p}$ involved in the analysis satisfy a similar kind of boundary value problem. The free surface condition, which is the most difficult task to satisfy, is of the same type for all cases, except that in the linear case $\left(\varphi^{(1)}, \varphi_{R j}^{(1) p}\right)$ it has homogeneous form (12, $59)$, and in the nonlinear $\left(\varphi^{(2)}, \varphi^{(3)}\right)$ or weakly nonlinear $\left(\varphi^{(11)}\right)$ cases it is nonhomogeneous one $(14,16,37)$. The same procedure will be used for each of these potentials. Thus, we define the following boundary value problem for the generic potential $\psi$ :

$$
\left.\begin{array}{ll}
\begin{array}{l}
\Delta \psi=0 \\
-\alpha \psi+\frac{\partial \psi}{\partial z}=Q(r, \theta)
\end{array} & z=0 \\
\frac{\partial \psi}{\partial n}=v(z, \theta) & \text { on thecy linders } \\
\frac{\partial \psi}{\partial n}=0 & z=-H \\
\text { radiation condition } & \mathrm{r} \rightarrow \infty
\end{array}\right\}
$$

For the sake of clarity, the potential $\psi$ is further subdivided in two parts $\psi=\psi_{B}+\psi_{Q}$ with the following BVP-s for each of them :

$$
\left.\begin{array}{ll}
\begin{array}{l}
\Delta \psi_{B}=0 \\
-\alpha \psi_{B}+\frac{\partial \psi_{B}}{\partial z}=Q(r, \theta)
\end{array} & 0 \geq z \geq-H \\
\frac{\partial \psi_{B}}{\partial n}=0 & \text { on thecy linders } \\
\frac{\partial \psi_{B}}{\partial n}=0 & z=-H \\
\lim \left[\sqrt{\mathrm{k}_{0} r}\left(\frac{\partial \psi_{B}}{\partial r}-i k_{0} \psi_{B}\right)\right]=0 & \mathrm{r} \rightarrow \infty
\end{array}\right\}
$$

$$
\begin{array}{ll}
\Delta \psi_{Q}=0 & 0 \geq z \geq-H \\
-\alpha \psi_{Q}+\frac{\partial \psi_{Q}}{\partial z}=Q(r, \theta) & z=0 \\
\frac{\partial \psi_{Q}}{\partial n}=0 & \text { on thecylin } \\
\frac{\partial \psi_{Q}}{\partial n}=0 & z=-H \\
\text { radiationcondition } & \mathrm{r} \rightarrow \infty
\end{array}
$$

The potential $\psi_{B}$ is the part of the potential caused by the imposed normal velocity on the body boundary, while the $\psi_{Q}$ is the part caused by the forcing on the free surface. These two parts are fundamentally different and will be treated separately.

In the case of small forward speed another kind of BCP should also be considered. In fact the potential $\bar{\phi}$ cannot be treated as a special case of potential $\psi$, and an independent BCP should be solved :

$$
\left.\begin{array}{ll}
\Delta \bar{\phi}=0 & 0 \geq z \geq-H \\
\frac{\partial \bar{\phi}}{\partial z}=0 & z=0 \\
\frac{\partial \bar{\phi}}{\partial z}=\frac{\partial x}{\partial n} & \text { on thecylinders } \\
\frac{\partial \bar{\phi}}{\partial z}=0 & z=-H \\
\frac{\partial \rightarrow 0}{\phi \rightarrow \infty} & r \rightarrow \infty
\end{array}\right\}
$$

This potential is of the local type and does not generate waves.

On the other hand, the cylinder configurations which will be treated here are :

\footnotetext{
- bottom mounted single cylinder

- $\quad$ single truncated cylinder

array of bottom mounted cylinders
}

We note here that the case of truncated cylinders array will not be treated explicitly but it can be constructed as a combination of the single truncated 
cylinder case and the case of bottom mounted cylinders array.

\subsection{Bottom Mounted Single Cylinder}

\subsubsection{Potential $\psi_{B}$}

The general methodology for this kind of potentials was first given in [12] for infinite water depth (2D of vertical wall for finite or infinite water depth was also considered). The method is the eigenfunction expansion which is possible due to the relatively simple geometry. We briefly resume the basic steps.

The solution is assumed in the following eigenfunction expansion form :

$\psi_{B}=\sum_{m=-\infty}^{\infty}\left[f_{0}(z) \beta_{m 0} H_{m}\left(k_{o} r\right)+\sum_{n=1}^{\infty} f_{n}(z) \beta_{m n} K_{m}\left(k_{n} r\right)\right] e^{i m \theta}$

where $H_{m}$ are Hankel functions of the first kind $H_{m}=J_{m}+i Y_{m}$ and $K_{m}$ are the modified Bessel functions. The functions $f_{n}(z)$ are defined by:

$$
f_{0}(z)=\frac{\cosh k_{0}(z+H)}{\cosh k_{o} H}, f_{n}(z)=\frac{\cos k_{n}(z+H)}{\cos k_{n} H}
$$

with $\alpha=k_{0} \tanh k_{0} H=-k_{n} \tan k_{n} H$.

Application of the boundary condition on the cylinder (69) and the use of orthogonality of eigenfunctions, gives the expressions for the unknown coefficients $\beta_{m n}$ :

$$
\begin{aligned}
& \beta_{m 0}=-\frac{2 C_{0}}{k_{0} H^{\prime}{ }_{m}\left(k_{0} a\right)} \int_{-H}^{0} f_{0}(z) v_{m}(z) d z \\
& \beta_{m n}=-\frac{2 C_{n}}{k_{0} H^{\prime}{ }_{m}\left(k_{0} a\right)} \int_{-H}^{0} f_{0}(z) v_{m}(z) d z
\end{aligned}
$$

where $C_{0}$ and $C_{n}$ are defined by :

$$
C_{0}=\left[2 \int_{-H}^{0} f_{0}^{2}(z) d z\right]^{-1}, C_{n}=\left[2 \int_{-H}^{0} f_{n}^{2}(z) d z\right]^{-1}
$$

and $v_{m}(z)$ follows from the Fourier series expansion :

$$
v(z)=\sum_{m=-\infty}^{\infty} v_{m}(z) e^{i m \theta}
$$

\subsubsection{Potential $\psi_{Q}$}

The situation is now more complicated and the eigenfunction expansion method alone is not sufficient. The integral equation method must be introduced. The method presented here is based on using the integral equation technique with the classical Green function expressed in its eigenfunction expansion for [13]. This method is inspired from the method presented in a relatively unnoticed (publication in Japanese) paper [21] where the interaction of vertical circular cylinder with water waves and small current was treated. We note here that two alternative methods are also possible [3, 24]. The first one uses the special kind of the Green function which satisfy the condition of zero normal velocity on the cylinder, while the second one uses the Weber transform technique. The final expressions are essentially the same. We describe now briefly the general method presented in [17]. First we write the Green function for two points, $\boldsymbol{x}=(x, y, z)=(r, \theta, z) \quad$ and $\quad \xi=(\xi, \eta, \zeta)=$ $(\rho, \vartheta, \zeta)$, is its eigenfunction expansion form :

$$
G(x, \xi)=\sum_{m=-\infty}^{\infty} G_{m}(r, z ; \rho, \zeta) e^{i m(\theta-\vartheta)}
$$

with :

$$
\begin{aligned}
& G_{m}(r, z ; \rho, \zeta) \\
& =-\frac{i}{2} C_{0}\left(\begin{array}{l}
H_{m}\left(k_{0} r\right) J_{m}\left(k_{0} \rho\right) \\
J_{m}\left(k_{0} r\right) H_{m}\left(k_{0} \rho\right)
\end{array}\right) f_{0}(z) f_{0}(\zeta) \\
& -\frac{1}{\pi} \sum_{n=1}^{\infty} C_{n}\left(\begin{array}{l}
K_{m}\left(k_{n} r\right) I_{m}\left(k_{n} \rho\right) \\
I_{m}\left(k_{n} r\right) K_{m}\left(k_{n} \rho\right)
\end{array}\right) f_{n}(z) f_{n}(\zeta) \\
& \qquad\left(\begin{array}{l}
r>\rho \\
r<\rho
\end{array}\right)
\end{aligned}
$$


The integral equation for the BVP (70) can be written in the form :

$$
\begin{aligned}
& \left(\begin{array}{c}
\psi_{Q}(x) \\
0
\end{array}\right)+\int_{S_{B 0}} \psi_{Q}(\xi) \frac{\partial G(x, \xi)}{\partial \rho} d S \\
& =-\int_{S_{F}} G(x, \xi) Q_{D}(\rho, \vartheta) d S \\
& \quad+\int_{S_{\infty}}\left[G(x, \zeta) \frac{\partial \psi_{Q}(\xi)}{\partial \rho}-\psi_{Q}(\xi) \frac{\partial G(x, \xi)}{\partial \rho}\right] d S \\
& \left.\qquad \begin{array}{l}
r>a \\
r<a
\end{array}\right)
\end{aligned}
$$

It can be shown that the integral over the surface at infinity $S_{\infty}$ disappears in all cases considered here [17], which represents, in some way, the radiation condition for $\psi_{Q}$.

The next step is to write the solution for $\psi_{Q}$ on the cylinder, in the eigenfunction expansion form :

$$
\begin{aligned}
\psi_{Q}(a, \theta, z) & =\sum_{-\infty}^{\infty} \psi_{Q m}(a, z) e^{i m \theta} \\
& =\sum_{-\infty}^{\infty}\left[f_{0}(z) A_{m 0}+\sum_{n=1}^{\infty} f_{n}(z) A_{m n}\right] e^{i m \theta}
\end{aligned}
$$

If we write now the integral equation for one point inside the cylinder, $r=a-\delta,(a \geq \delta>0)$ we can deduce the value of the $A_{m n}$ coefficients by using the orthogonal property of eigenfunctions :

$$
\begin{gathered}
A_{m 0}=-\frac{2 C_{0} \int_{a}^{\infty} H_{m}\left(k_{0} \rho\right) Q_{D m}(\rho) \rho d \rho}{k_{0} a H_{m}^{\prime}\left(k_{0} a\right)}, \\
A_{m n}=-\frac{2 C_{n} \int_{a}^{\infty} K_{m}\left(k_{n} \rho\right) Q_{D m}(\rho) \rho d \rho}{k_{n} a K_{m}^{\prime}\left(k_{n} a\right)}
\end{gathered}
$$

By returning this solution in the integral equation we obtain the expression for each Fourier mode of the potential at any point in the fluid :

$$
\begin{aligned}
\psi_{Q m}(r, z)= & \pi i C_{0} f_{0}(z) H_{m}\left(k_{0} r\right) \\
& \times \int_{a}^{r}\left[J_{m}\left(k_{0} \rho\right)-Z_{m 0} H_{m}\left(k_{0} \rho\right)\right] Q_{D m}(\rho) \rho d \rho \\
& +2 \sum_{n=1}^{\infty} C_{n} f_{n}(z) K_{m}\left(k_{n} r\right) \\
& \times \int_{a}^{r}\left[I_{m}\left(k_{n} \rho\right)-z_{m n} K_{m}\left(k_{n} \rho\right)\right] Q_{D m}(\rho) \rho d \rho \\
& +\pi i C_{0} f_{0}(z)\left[J_{m}\left(k_{0} r\right)-Z_{m 0} H_{m}\left(k_{0} r\right)\right] \\
& \times \int_{r}^{\infty} H_{m}\left(k_{0} \rho\right) Q_{D m}(\rho) \rho d \rho \\
& +2 \sum_{n-1}^{\infty} C_{n} f_{n}(z)\left[I_{m}\left(k_{n} r\right)-Z_{m n} K_{m}\left(k_{n} r\right)\right] \\
& \times \int_{r}^{\infty} K_{m}\left(k_{n} \rho\right) Q_{D m}(\rho) \rho d \rho
\end{aligned}
$$

where :

$$
Z_{m 0}=\frac{J_{m}^{\prime}\left(k_{0} a\right)}{H^{\prime}{ }_{m}\left(k_{0} a\right)}, Z_{m n}=\frac{I_{m}^{\prime}\left(k_{n} a\right)}{K_{m}^{\prime}\left(k_{n} a\right)}
$$

As we can see, we represented the solution, of the BVP with nonhomogeneous free surface condition, as a series of eigenfunctions which individually satisfies the homogeneous free surface condition. However it can be shown [3] that this series satisfies an nonhomogeneous condition in the limiting sense $z \rightarrow 0^{-}$.

This will cause the appearance of a logarithmic type of singularity when calculating the potential on the free surface. Another problem arises in the calculation of the infinite integrals in (83) which generally include slowly decaying functions so that the asymptotic methods should be involved. In summary, we should be very careful $[3,17]$ when evaluating the different integrals and infinite sums involved in the above expressions.

\subsubsection{Potential $\bar{\phi}$}

In the case of the bottom mounted vertical circular cylinder, the expression for the double body potential $\bar{\phi}$ is very simple, and is represented by the dipole potential :

$\bar{\phi}=-\frac{a^{2}}{r} \cos \theta$

\subsection{Single Truncated Cylinder}

The configuration and the notations for this case are presented on the figure 2 . 


\subsubsection{Potential $\psi_{B}$}

First we consider the diffraction case which means that $v(z)$ becomes the radial derivative of the incident potential denoted here by $\psi^{I}$. The method used here is inspired from [10], and is also based on the use of eigenfunction expansions in respective domains. The eigenfunction expansion for the interior region can be written in the form :

$$
\begin{aligned}
\psi^{i n} & =\sum_{m=-\infty}^{\infty}\left[b_{m 0}^{\psi}\left(\frac{r}{a}\right)^{|m|}\right. \\
& \left.+\sum_{n=1}^{\infty} b_{m n}^{\psi} \cos \lambda_{n}(z+H) I_{m}\left(\lambda_{n} r\right)\right] e^{i m \theta}
\end{aligned}
$$

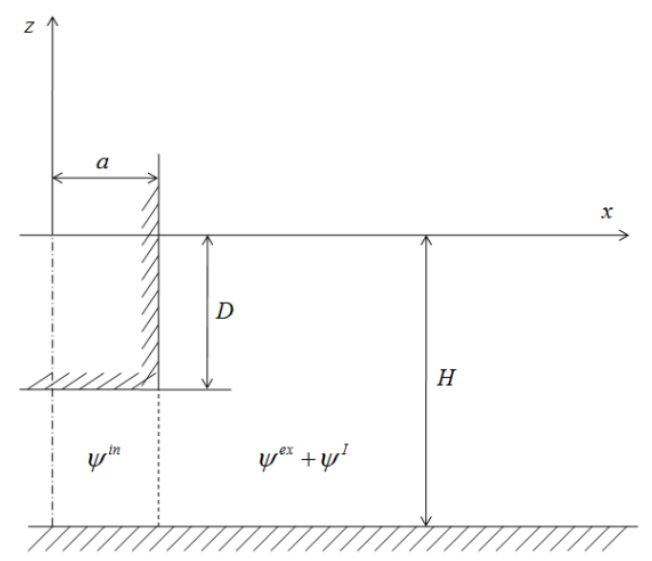

Fig. 2. Different fluid domains.

with $\lambda_{n}=n \pi /(H-D)$, and $I_{m}$ denoting the modified Bessel functions.

In the exterior domain we write the eigenfunction expansion in the form similar to (72) :

$$
\begin{aligned}
\psi^{e x}= & \sum_{m=-\infty}^{\infty}\left[c_{m 0}^{\psi} f_{0}(z) H_{m}\left(k_{0} r\right)\right. \\
& \left.+\sum_{n=1}^{\infty} c_{m n}^{\psi} f_{n}(z) K_{m}\left(k_{n} r\right)\right] e^{i m \theta}
\end{aligned}
$$

Orthogonality of the eigenfunctions in $z$ direction is now used, and the boundary conditions on the cylinder $(r=a, 0 \geq z \leq-D)$ and continuity conditions on the intersection surface $(r=$ $a,-D \geq z \leq-H)$ are written in the form :

$$
\int_{-H}^{-D}\left(\psi^{e x}+\psi^{I}\right) d z=\int_{-H}^{-D} \psi^{i n} d z
$$

$$
\begin{aligned}
& \int_{-H}^{0} \frac{\partial}{\partial r}\left(\psi^{e x}+\psi^{I}\right) f_{n}(z) d z \\
&= \int_{-H}^{-D} \frac{\partial \psi^{i n}}{\partial r} f_{n}(z) d z \quad n=1, \infty \\
& \int_{-H}^{0} \frac{\partial}{\partial r}\left(\psi^{e x}+\psi^{I}\right) f_{0}(z) d z=\int_{-H}^{-D} \frac{\partial \psi^{i n}}{\partial r} f_{0}(z) d z \\
& \quad \int_{-H}^{-D}\left(\psi^{e x}+\psi^{I}\right) \cos \lambda_{n}(z+H) d z \\
&=\int_{-H}^{-D} \psi^{i n} \cos \lambda_{n}(z+H) d z \quad n=1, \infty
\end{aligned}
$$

We can now write the linear system of equations for each Fourier mode $m=-\infty, \infty$ :

$$
\begin{aligned}
& b_{m 0}^{\psi}=c_{m 0}^{\psi} D_{00}^{m \psi}+\sum_{n=1}^{\infty} c_{m n}^{\psi} D_{0 n}^{m \psi}+F_{0}^{m \psi} \\
& b_{m l}^{\psi}=c_{m 0}^{\psi} D_{l 0}^{m \psi}+\sum_{n=1}^{\infty} c_{m n}^{\psi} D_{l \mathrm{n}}^{m \psi}+F_{l}^{m \psi} \quad l=1, \infty \\
& c_{m 0}^{\psi}=b_{m 0}^{\psi} E_{00}^{m \psi}+\sum_{n=1}^{\infty} b_{m n}^{\psi} E_{0 n}^{m \psi}+G_{0}^{m \psi} \\
& c_{m l}^{\psi}=b_{m 0}^{\psi} E_{l 0}^{m \psi}+\sum_{n=1}^{\infty} b_{m n}^{\psi} E_{l \mathrm{n}}^{m \psi}+G_{l}^{m \psi} \quad l=1, \infty
\end{aligned}
$$

which can be rewritten in the matrix form :

$$
\left(\begin{array}{cc}
\mathrm{I} & -\mathrm{D}^{m \psi} \\
-\mathrm{E}^{m \psi} & \mathrm{I}
\end{array}\right)\left(\begin{array}{l}
\mathrm{B}_{m}^{\psi} \\
\mathrm{C}_{m}^{\psi}
\end{array}\right)=\left(\begin{array}{l}
\mathrm{F}^{m \mu} \\
\mathrm{G}^{m \psi}
\end{array}\right)
$$

with

$$
\begin{aligned}
& \mathbf{D}^{m \psi}=\left(\begin{array}{ccc}
D_{00}^{m \psi} & \cdots & D_{0 n}^{m \psi} \\
\cdots & \cdots & \cdots \\
D_{l 0}^{m \psi} & \cdots & D_{l n}^{m \psi}
\end{array}\right) \\
& \mathbf{E}^{m \psi}=\left(\begin{array}{ccc}
E_{00}^{m \psi} & \cdots & E_{0 n}^{m \psi} \\
\cdots & \cdots & \cdots \\
E_{l 0}^{m \psi} & \cdots & E_{l n}^{m \psi}
\end{array}\right) \\
& \mathbf{B}_{m}^{\psi}=\left[b_{m 0}^{\psi}, b_{m 1}^{\psi}, \cdots, b_{m n}^{\psi}\right]^{T} \mathbf{C}_{m}^{\psi}=\left[c_{m 0}^{\psi}, c_{m 1}^{\psi}, \cdots, c_{m n}^{\psi}\right]^{T} \\
& \mathbf{F}^{m \psi}=\left[F_{0}^{m \psi}, F_{1}^{m \psi}, \cdots, F_{n}^{m \psi}\right]^{T}, \\
& \mathbf{G}^{m \psi}=\left[G_{0}^{m \psi}, G_{1}^{m \psi}, \cdots, G_{n}^{m \psi}\right]^{T}
\end{aligned}
$$


and I denoting the unity matrix.

All the coefficients in the matrix equation above are easily deduced from (88) to (91). The solution of the system gives the unknown coefficients $b_{m n}^{\psi}$ and $c_{m n}^{\psi}$ which terminates the calculation of the potential. In the case of radiation the procedure is similar except that particular solution should be added to the expression for the potential in the interior region : the eigenfunction expansion (86) satisfies the condition of zero normal velocity on the bottom of the cylinder which is not the case for all radiation problems.

Only three independent radiation problems need to be considered.

\section{- Surge $j=1$}

The boundary conditions for surge are :

$\partial \varphi_{R 1}^{(1) i n} / \partial z=0, \quad z=-D, a \geq r \geq 0$

$\partial \varphi_{R 1}^{(1) i n} / \partial r=\cos \theta, \quad r=a, 0 \geq z \geq-D$

so that there is no need for additional particular solution but only the modes $\mathrm{m}=1$ and $\mathrm{m}=-1$ in the expansions $(86,72)$ should be included.

- Heave $j=3$

The boundary conditions for heave are :

$\partial \varphi_{R 3}^{(1) i n} / \partial z=1, \quad z=-D, a \geq r \geq 0$

$\partial \varphi_{R 3}^{(1) i n} / \partial r=0, \quad r=a, 0 \geq z \geq-D$

so that the particular solution can be chosen as :

$\varphi_{R 3}^{(1) i n}=-\frac{1}{2 D}\left(z^{2}-\frac{r^{2}}{2}\right)$

and only the zeroth Fourier mode is included in the expansions $(86,72)$

\section{$\operatorname{Pitch} j=5$}

The boundary conditions for pitch are :

$\partial \varphi_{R 5}^{(1) i n} / \partial z=-r \cos \theta \quad, \quad z=-D, a \geq r \geq 0$

$\partial \varphi_{R 3}^{(1) i n} / \partial r=0, \quad r=a, 0 \geq z \geq-D$

so that the particular solution becomes :
$\varphi_{R 5}^{(1) i n}=\frac{r}{2 D}\left(z^{2}-\frac{r^{2}}{4}\right) \cos \theta$

and only the modes $\mathrm{m}=1$ and $\mathrm{m}=-1$ in the expansions $(86,72)$ should be included.

A part of that, the procedure is completely similar and will not be detailed here.

\subsubsection{Potential $\psi_{Q}$}

The method remains very similar. In fact, the solution $\psi_{Q}$ for the complete cylinder $(81,83)$, plays here the role of the incident potential $\psi^{I}$. All expressions and general methodology leading to the linear system of equations are exactly the same. However, the case of $\varphi_{p}^{(11)}$ is a little bit different, In fact, it is simpler since the particular solution is explicitly known and can be used directly as the incident potential $\psi^{I}$ for the homogeneous part [15].

\subsubsection{Potential $\bar{\phi}$}

Even if the methodology remains the same for this case, the eigenfunctions expansion in the exterior region changes due to the different free surface condition :

$$
\begin{aligned}
\bar{\phi}^{e x} & =\sum_{m=-\infty}^{\infty}\left[c_{m 0}^{\bar{\phi}}\left(\frac{a}{r}\right)^{|m|}\right. \\
& \left.+\sum_{n=1}^{\infty} c_{m n}^{\bar{\phi}} \cos \mu_{n}(z+H) K_{m}\left(\mu_{n} r\right)\right] e^{i m \theta}
\end{aligned}
$$

with $\mu_{n}=n \pi / H$.

The rest of the procedure is the same as in the case of $\psi_{B}$.

\subsection{Array of Bottom Mounted Cylinders}

The geometry and basic definitions are shown on the figure 3 .

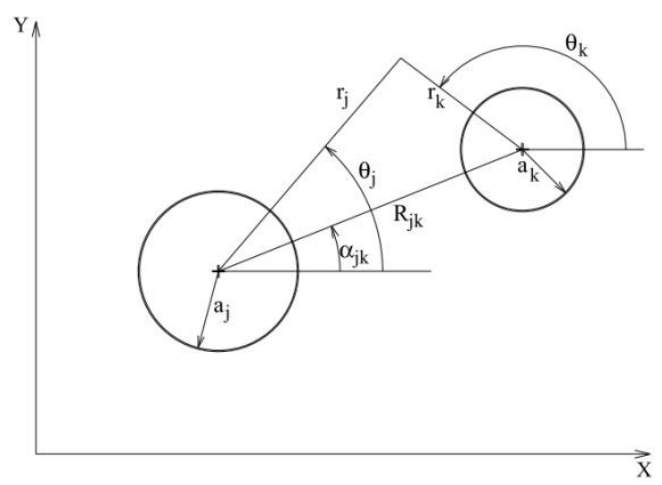

Fig. 3. Basic configuration. 


\subsubsection{Potential $\psi_{B}$}

The method which will be explained here is essentially based on the work by Linton \& Evans [14] where the linear diffraction by an array of bottom mounted cylinders was presented.

First we assume the following eigenfunction expansion for $\psi_{B}$ :

$$
\begin{aligned}
\psi_{B}= & \sum_{k=1}^{N_{c}} \sum_{m=-\infty}^{\infty}\left[f_{0}(z) D_{m 0}^{k} Z_{m 0}^{k} H_{m}\left(k_{0} r_{k}\right)\right. \\
& \left.+\sum_{l=1}^{\infty} f_{l}(z) D_{m l}^{k} Z_{m l}^{k} K_{m}\left(k_{l} r_{k}\right)\right] e^{i m \theta_{k}}
\end{aligned}
$$

After applying the boundary condition on each cylinder using the Graff's addition theorem for Bessel functions, we obtain the following systems of equations for the unknown coefficients $D_{m i}^{k}$ :

$$
D_{m 0}^{k}+\sum_{j \neq k} \sum_{n=-\infty}^{\infty} D_{n 0}^{j} Z_{n 0}^{j} e^{i(n-m) \alpha_{j k}} H_{n-m}\left(k_{0} R_{j k}\right)=\gamma_{m 0}^{k}
$$

$D_{m l}^{k}+(-1)^{m} \sum_{j \neq k} \sum_{n=-\infty}^{\infty} D_{n l}^{j} Z_{n l}^{j} e^{i(n-m) \alpha_{j k}} K_{n-m}\left(k_{l} R_{j k}\right)=\gamma_{m l}^{k}$

$m=-\infty, \infty, k=1, N_{c}, l=1, \infty$

where

$\gamma_{m 0}^{k}=\frac{2 C_{0}}{k_{0} J_{m}^{\prime}\left(k_{0} a_{k}\right)} \int_{-H}^{0} f_{0}(z) v_{m}(z) d z$,

$\gamma_{m l}^{k}=\frac{2 C_{l}}{k_{l} I_{m}^{\prime}\left(k_{l} a_{k}\right)} \int_{-H}^{0} f_{l}(z) v_{m}(z) d z$

Near the cylinder $k, r_{k}<R_{j k}, \forall j \neq k$, this expression can be simplified using the Graff's addition theorem together with $(110,111)$. The simple expression in terms of the $k$-th cylinder local coordinates is :

$$
\begin{aligned}
\psi_{B}\left(r_{k}, \theta_{k}, z\right) & =\sum_{m=-\infty}^{\infty}\left\{f _ { 0 } ( z ) \left[D_{m 0}^{k} Z_{m 0}^{k} H_{m}\left(k_{0} r_{k}\right)\right.\right. \\
& \left.+\left(\gamma_{m 0}^{k}-D_{m 0}^{k}\right) J_{m}\left(k_{0} r_{k}\right)\right] \\
& +\sum_{l=1}^{\infty} f_{l}(z)\left[D_{m l}^{k} Z_{m l}^{k} K_{m}\left(k_{l} r_{k}\right)\right. \\
& \left.\left.+\left(\gamma_{m l}^{k}-D_{m l}^{k}\right) I_{m}\left(k_{l} r_{k}\right)\right]\right\} e^{i m \theta_{k}}
\end{aligned}
$$

\subsubsection{Potential $\psi_{Q}$}

As in the single cylinder case we start by writing Green's theorem for one point outside the fluid domain :

$$
\iint_{S_{B}} \psi_{Q} \frac{\partial G}{\partial n} d S=\iint_{S_{F}} G Q d S
$$

The potential on the cylinder $k$ is then assumed in the form :

$$
\psi_{Q}^{k}\left(a_{k}, \theta_{k}, z\right)=\sum_{m=-\infty}^{\infty}\left[B_{m 0}^{k} f_{0}(z)+\sum_{l=1}^{\infty} B_{m l}^{k} f_{l}(z)\right] e^{i m \theta_{k}}
$$

After writing the Green's theorem (114) for one point inside the cylinder $k \rightarrow\left(r_{k}=a_{k}-\delta, 0<\right.$ $\delta \leq a_{k}$ ), carrying out the integration by $\zeta$, using the orthogonality of the functions $f_{n}(z)$ and the Graff's addition theorem for Bessel functions, exploiting the orthogonality of functions $e^{i m \theta}$ and rearranging the different terms we obtain the following systems of equations for the unknown coefficients $B_{m i}^{k}$ :

$$
\begin{aligned}
& B_{m 0}^{k}+\sum_{j \neq k} \frac{a_{j}}{a_{k}} \sum_{n=-\infty}^{\infty} B_{n 0}^{j} \frac{J_{n}^{\prime}\left(k_{0} a_{j}\right)}{H^{\prime}{ }_{m}\left(k_{0} a_{j}\right)} H_{n-m}\left(k_{0} R_{j k}\right) e^{i(n-m) \alpha_{j k}}= \\
& -\frac{C_{0}}{\pi a_{k} k_{0} H^{\prime}{ }_{m}\left(k_{0} a_{k}\right)} \iint_{S_{F}} H_{m}\left(k_{0} \rho_{k}\right) e^{-i m \vartheta_{k}} Q\left(\rho_{k}, \vartheta_{k}\right) d S
\end{aligned}
$$

$$
\begin{aligned}
& B_{m l}^{k}+(-1)^{m} \sum_{j \neq k} \frac{a_{j}}{a_{k}} \sum_{n=-\infty}^{\infty} B_{n l}^{j} \frac{I_{n}^{\prime}\left(k_{l} a_{j}\right)}{K^{\prime}{ }_{m}\left(k_{l} a_{j}\right)} K_{n-m}\left(k_{l} R_{j k}\right) e^{i(n-m) \alpha_{j k}}= \\
& -\frac{C_{l}}{\pi a_{k} k_{l} K_{m}^{\prime}\left(k_{l} a_{k}\right)} \iint_{S_{F}} K_{m}\left(k_{l} \rho_{k}\right) e^{-i m \vartheta_{k}} Q\left(\rho_{k}, \vartheta_{k}\right) d S
\end{aligned}
$$

$m=-\infty, \infty, k=1, N_{c}, l=1, \infty$

This completes the solution for the potential $\psi_{Q}$ on the cylinders.

Once the potential on the cylinders is found, the potential at any point in the fluid can be calculated using Green's theorem. The resulting expression is :

$$
\begin{aligned}
\psi_{Q}= & \sum_{j=1}^{N_{e}} 2 \pi a_{j} \sum_{m=-\infty}^{\infty}\left[B_{m 0}^{j} \frac{i k_{0}}{4} J_{m}^{\prime}\left(k_{0} a_{j}\right) f_{0}(z) H_{m}\left(k_{0} r_{j}\right)\right. \\
& \left.+\sum_{n=1}^{\infty} B_{m n}^{j} \frac{k_{n}}{2 \pi} I_{m}^{\prime}\left(k_{n} a_{j}\right) f_{n}(z) K_{m}\left(k_{n} r_{j}\right)\right] e^{i m \theta_{j}} \\
& -\iint_{S_{F}} G Q d S
\end{aligned}
$$


As in the case of $\psi_{B}$ this expression can be simplified near the cylinders $\left(r_{k}<R_{j k}, \forall j\right)$ :

$$
\begin{aligned}
\psi_{Q} & =\sum_{m=-\infty}^{\infty}\left\{\frac { i \pi a _ { k } k _ { 0 } } { 2 } f _ { 0 } ( z ) B _ { m 0 } ^ { k } \left[J_{m}^{\prime}\left(k_{0} r_{k}\right)\right.\right. \\
& \left.-H_{m}^{\prime}\left(k_{0} a_{k}\right) J_{m}\left(k_{0} r_{k}\right)\right] \\
& +\sum_{l=1}^{\infty} a_{k} k_{l} f_{l}(z) B_{m l}^{k}\left[I_{m}^{\prime}\left(k_{l} a_{k}\right) K_{m}\left(k_{l} r_{k}\right)\right. \\
& \left.-K_{m}^{\prime}\left(k_{l} a_{k}\right) I_{m}\left(k_{l} r_{k}\right)\right] \\
& +\int_{0}^{r_{k}}\left\{i \pi C _ { 0 } f _ { 0 } ( z ) \left[H_{m}\left(k_{0} r_{k}\right)\right.\right. \\
& \left.-J_{m}\left(k_{0} r_{k}\right) H_{m}\left(k_{0} \rho_{k}\right)\right] \\
& +2 \sum_{l=1}^{\infty} C_{l} f_{l}(z)\left[K_{m}\left(k_{l} r_{k}\right) I_{m}\left(k_{l} \rho_{k}\right)\right. \\
& \left.\left.\left.-I_{m}\left(k_{l} r_{k}\right) K_{m}\left(k_{l} \rho_{k}\right)\right]\right\} Q_{m}\left(\rho_{k}\right) \rho_{k} d \rho_{k}\right\} e^{i m \theta_{k}}
\end{aligned}
$$

For $r_{k}=a_{k}$ expression reduces to (115).

The numerical implementation of the method must be performed very carefully because of many convergence problems associated with the eigenfunction expansions, Graff's theorem and especially numerical integration over the free surface. For details we refer to [19].

\section{Results and Discussions}

We start by presenting some results for the exciting forces on a fixed bottom mounted cylinder, up to third order. On figures 4,5 and 6, first, second and third order exciting surge forces are presented for the cylinder of the radius $a$ standing in the water of the depth $H=10 a$.

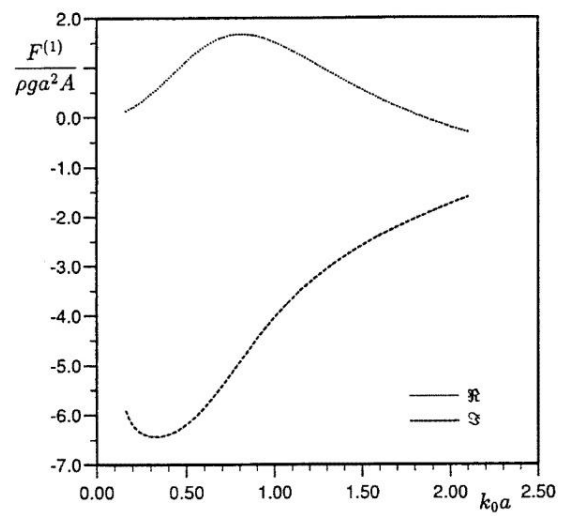

Fig. 4. Real and imaginary part of the first order force on the bottom mounted cylinder of radius $a$ in the water depth $H=$ $10 a$.
As usual, the total second and third order forces are decomposed into different components associated with the different contributions :

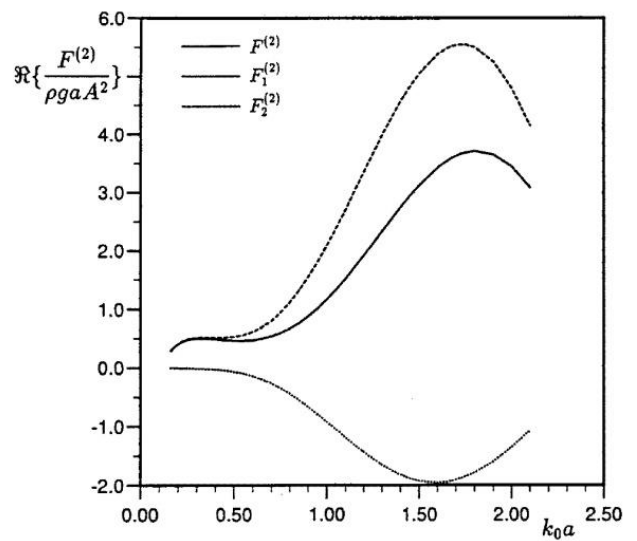

(a)

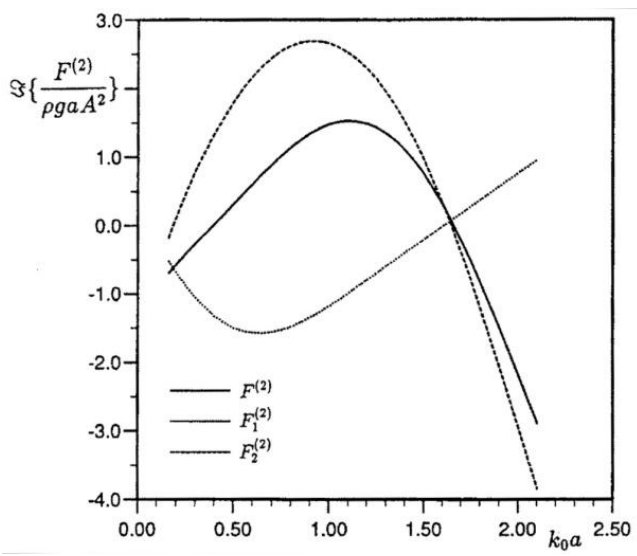

(b)

Fig. 5. Real (a) and imaginary (b) part of the second order force on the bottom mounted cylinder of radius $a$ in the water depth $H=10 a$. 


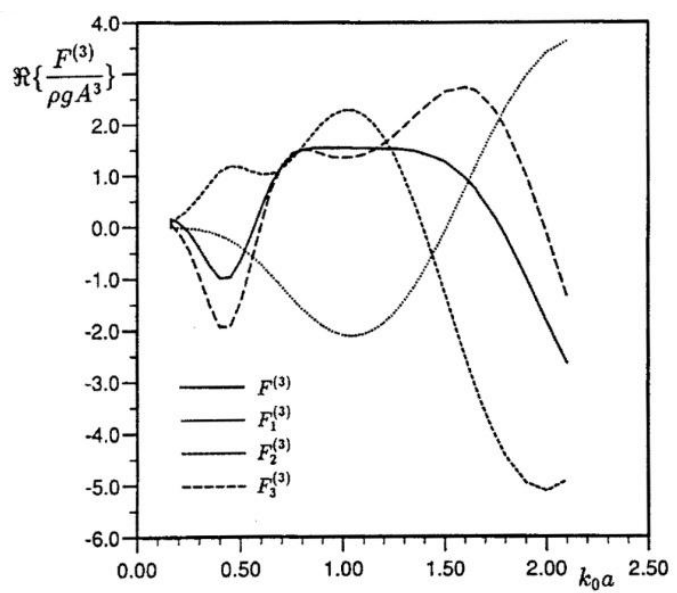

(a)

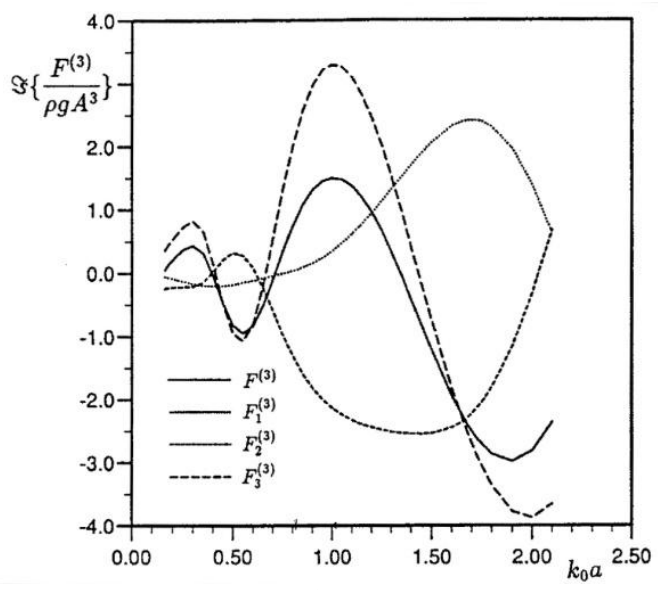

(b)

Fig. 6. Real (a) and imaginary (b) part of the third order force on the bottom mounted cylinder of radius $a$ in the water depth $H=10 a$.

- $\quad F_{1}^{(2)}$ - part of the second order force induced by the quadratic products of the first order quantities

- $F_{2}^{(2)}$ - part of the second order force induced by the second order potential

- $F^{(2)}=F_{1}^{(2)}+F_{2}^{(2)}$

- $F_{1}^{(3)}$ - part of the third order force induced by the triple products of the first order quantities

- $\quad F_{2}^{(3)}$ - part of the third order force induced by the products of the first and second order quantities

- $\quad F_{3}^{(3)}$ - part of the third order force induced by the third order potential

- $F^{(3)}=F_{1}^{(3)}+F_{2}^{(3)}+F_{3}^{(3)}$
From a practical point of view, a disappointing result shown by these figures is that all contributions to the forces are important in the whole practical range of frequencies and none of them can be neglected.

As a consequence, $\mathrm{u}$ approximative methods based on long wave theories have a very restricted range of application and often have no practical utility [17].

We turn now to the problem with small forward speed and first we show the results for the first order surge exciting forces on a bottom mounted cylinder, in a different water depth, $H=a$.

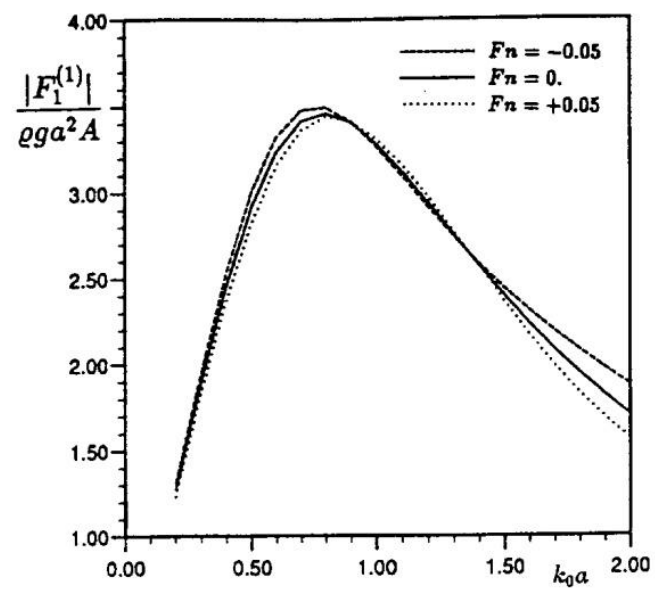

Fig. 7. First order surge exciting force on the bottom mounted cylinder for three different values of Froude number. Cylinder has radius $a$, water depth is $H=10 a$ and wave incidence is $\beta$ $=0$.

On figure 7 this force is presented for three different values of the Froude number $F_{n}=U / \sqrt{g a}$. The added mass and damping coefficients for the same case are presented on figure 8 . Interesting results concerning the wave drift damping coefficients are shown on figures 9 and 10. Figure 9 concerns the case of the fixed cylinder, and in this case the results obtained by the simple formula (46) are indistinguishable from the semi-analytical calculations. Contrarily, the case of the freely floating cylinder presented on the figure 10 leads to a different conclusion because there are important differences between the two sets of results. Surprisingly the case of $\beta=\pi / 4$ shows again complete agreement between the simple formula and the analytical calculations!? We note that the calculations of the wave drift damping coefficient were performed both by the far-field method (45) and the near-field method (not presented here) and that 


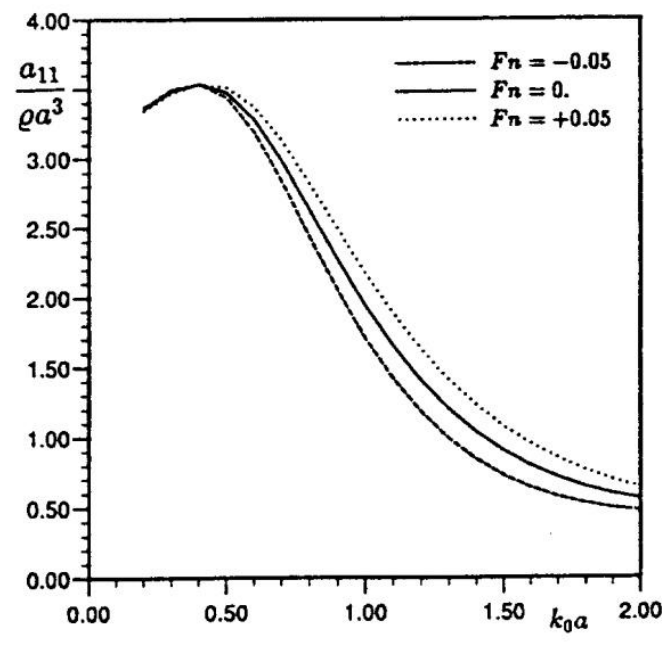

(a)

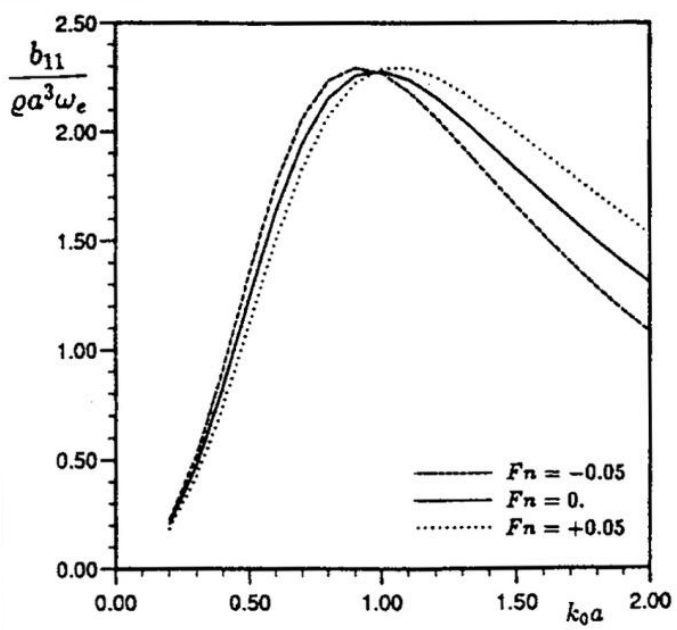

(b)

Fig. 8. Added mass (a) and damping (b) coefficients for the bottom mounted cylinder and for three different values of Froude number. Cylinder has radius $a$, water depth is $H=a$ and wave incidence is $\beta=0$.

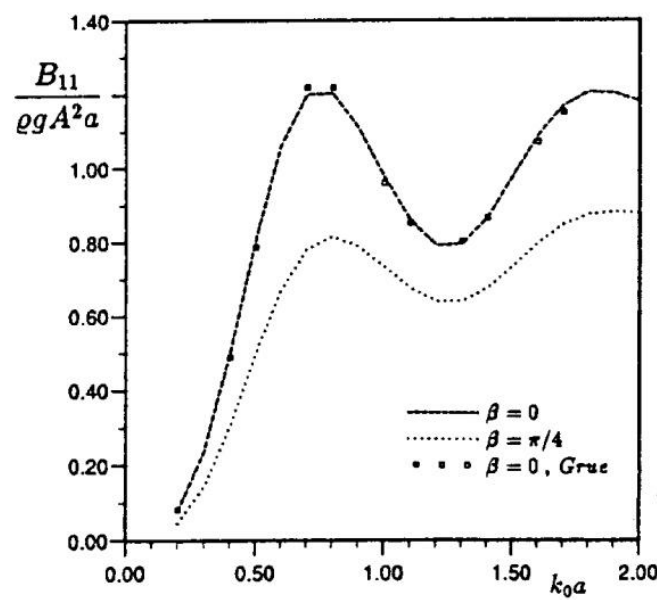

Fig. 9. Wave drift damping coefficient $B_{11}$ for a fixed bottom mounted cylinder. Cylinder has radius $a$ and water depth is $H$ $=a$.

both sets of results are identical. Additionaly, the completely numerical results obtained by Grue [11] also agree with these analytical results.

In figure 11, we present now one result useful for more detailed benchmarking purposes. It concerns the free surface wave elevation around the cylinder. The cylinder is from the previous case and wave number is $k_{0}=1.2$. We note important modifications of the free surface influenced by the presence of the current, which has a direct consequence on severe modifications of the steady second order loads. 


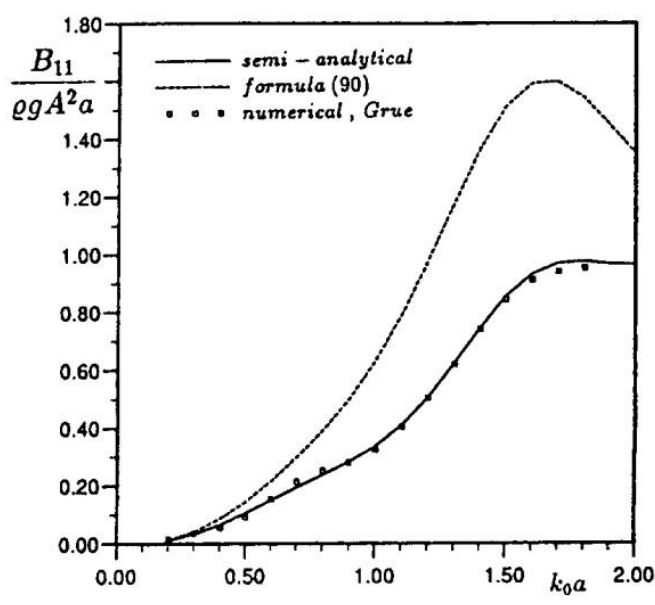

(a)

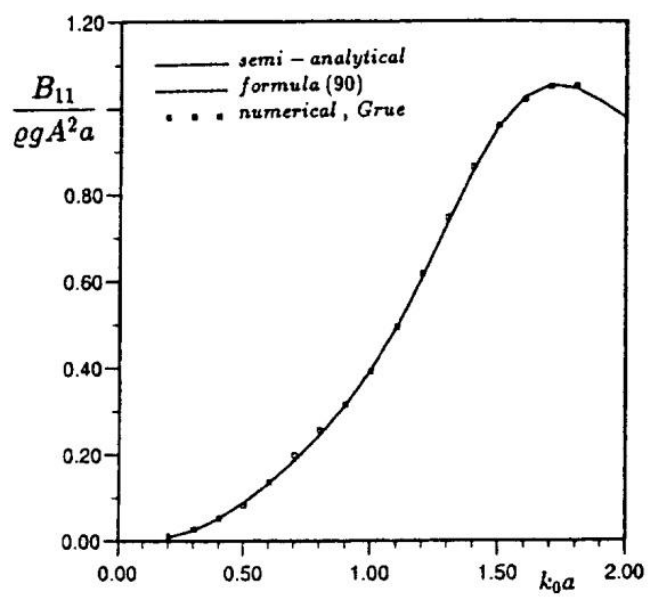

(b)

Fig. 10. Wave drift damping coefficient $B_{11}$ for freely floating complete cylinder and for incidences $\beta=0$ (a) and $\beta=\pi / 4$ (b). Cylinder has radius $a$ and water depth is $H=a$.

Another interesting phenomenon concerning the wave-current-floating body interactions is the secularity (unphysical growth of the solution far away from the body) of the additional perturbation by $\tau$ (35). In spite of the recognized secularity of the solution the perturbation by $\tau$ is a common approach to treat this problem [26]. Only recently [25, 5] it was numerically shown that both solutions (secular and non-secular) are the same as far as the global forces are concerned. This was further confirmed by the multiple scale approach [18] and it was shown theoretically that two solutions should be the same on the body. However, two solutions differ very quickly when we leave the body so that

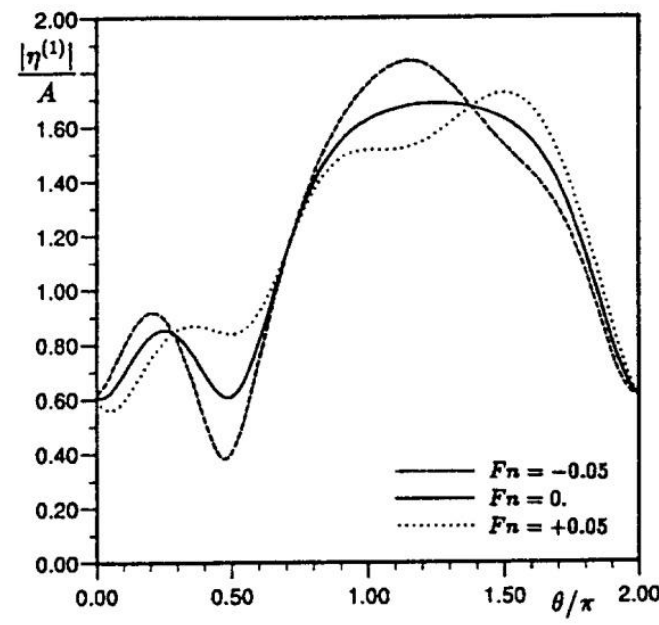

Fig. 11 Free surface wave elevation for the fixed bottom mounted cylinder. Cylinder has radius $a$, water depth is $H=a$, wave incidence is $\beta=\pi / 4$ and wave number $k_{0} a=1.2$.

the secular approach should not be used in the calculation of the wave elevation near the body (between the columns of a multi column structure for example) because the elevation can be seriously overestimated. Figure 12 which represents the view of the potential (proportional to the wave elevation) on the free surface around the fixed cylinder confirms the above comments.

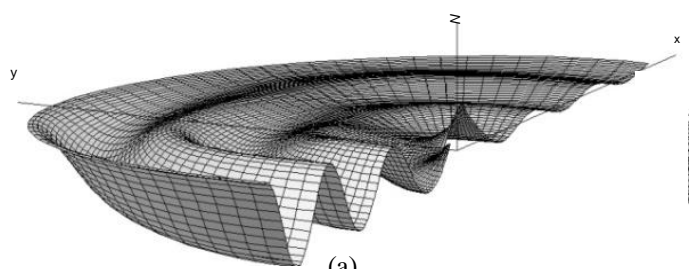

(a)

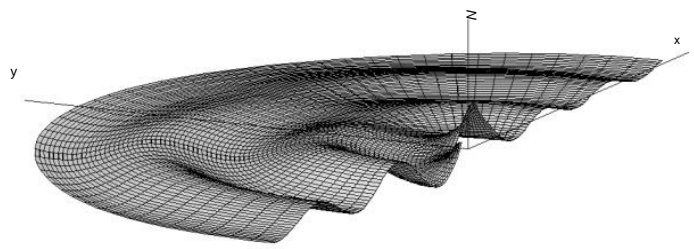

(b)

Fig. 12. Secular (a) and non-secular (b) solution for the imaginary part of the diffracted potential on the free surface. Cylinder radius is $a=1$., the water depth is $H=a$, wave incidence is $\beta=0$ and wavenumber is $k_{0}=0.7$.

Now we turn to the case of the truncated cylinder and in figure 13 we first present the values of the first order surge and heave exciting forces for different draughts of the cylinder with the radius $a$ in 


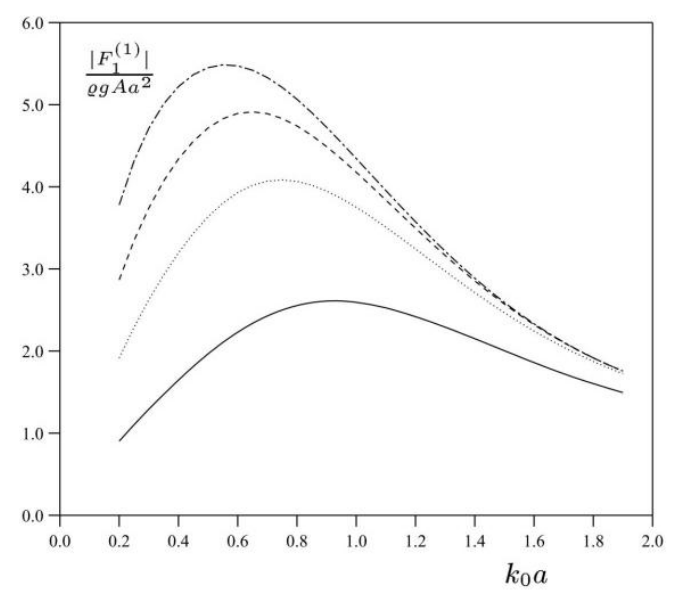

(a)

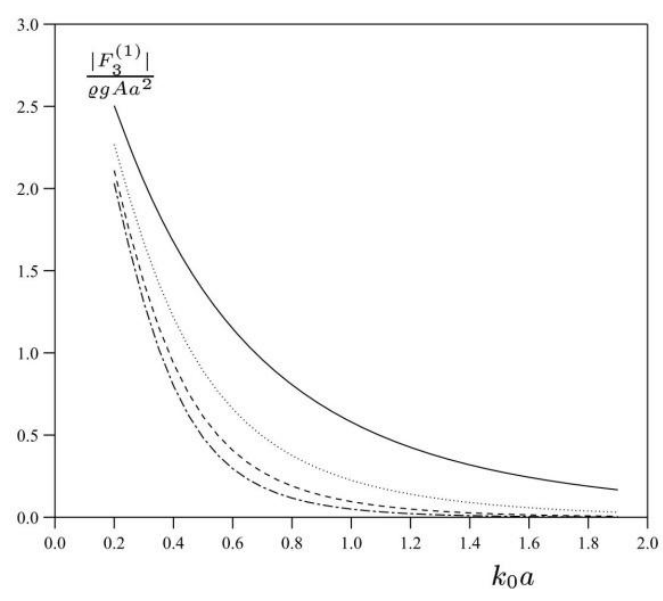

(b)

Fig. 13. First order surge (a) and heave (b) exciting forces on bottom mounted vertical cylinder. The cylinder radius is $a$, water depth is $H=5 a$ and wave incidence is $\beta=0$. Dott dashed line : $D=4 a$; dashed line: $D=3 a$; dotted line $D=2 a$; full line $D=a$.

the water of depth $5 a$. More interesting results are shown in figure 14. It concerns the comparisons of the wave drift damping coefficient calculated by the simple formula with the values obtained according to the semi-analytical method described here. It can be seen that the formula works quite well for deep-draught cylinder but not for the "short" one. Contrarily to the case of the freely floating bottom mounted cylinder, noticeable differences exist between the formula and semi-analytical calculations also for $\beta=\pi / 4$.

We consider now the case of an array of bottom mounted circular cylinders. In figure 15 we show again the results for the wave drift damping coeffi- cient. In this case, the theory and the formula give exactly the same results. The most complicated results obtained by the semi-analytical approach described here, are pre presented in last figures 16, 17. They concern the values of the free surface elevation up to the second order for an array of cylinders. As for the forces on the figure 5, we use the equivalent notations for the free surface elevations. It is believed that the second order theory can explain wave amplifications around off-shore structures which are not captured by linear theory. In some extent this is confirmed by these figures where we can see that in some cases, the second order component of the free surface elevation can dominate the first order one. The numerical complexities in the evaluation of these quantities are numerous, however the semi-analytical method remains superior to the complete numerical models because of its rapidity and precision.

Finally we consider the problem of hydroelasticity of the vertical column. The case chosen to show the efficiency of the method is that from [23]. The cylinder has a radius of $10 \mathrm{~m}$, in water of depth $200 \mathrm{~m}$. The distributed mass along the length of the cylinder is assumed to be half of its displaced mass. A concentrated mass $m_{0}$ equal to the total displaced mass (twice the distributed mass) is located at the free surface. The stiffness factor of the cylinder is chosen such that $E I / H^{3}=0.41 m_{0} s^{-2}$. The amplitude of the displacement at the top of the column for different wave periods is shown in figure $18 a$. We can observe the highly tuned resonance at approximately $T=6.5 \mathrm{~s}$. Finally, in figure $18 b$ we present the deformation of the column for $T=6.5 \mathrm{~s}$, in an instant when the top of the column reaches its maximal displacement. 


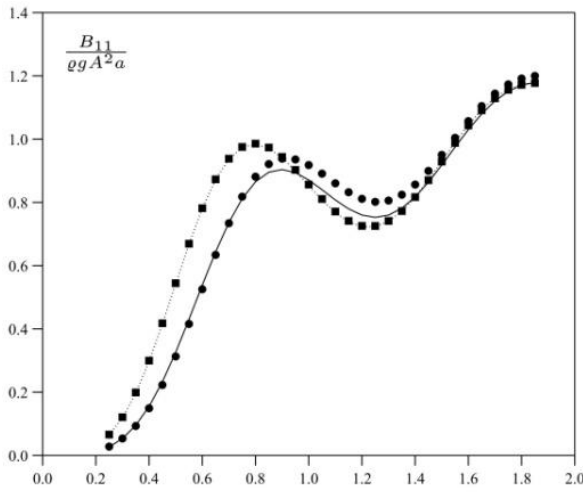

(a)

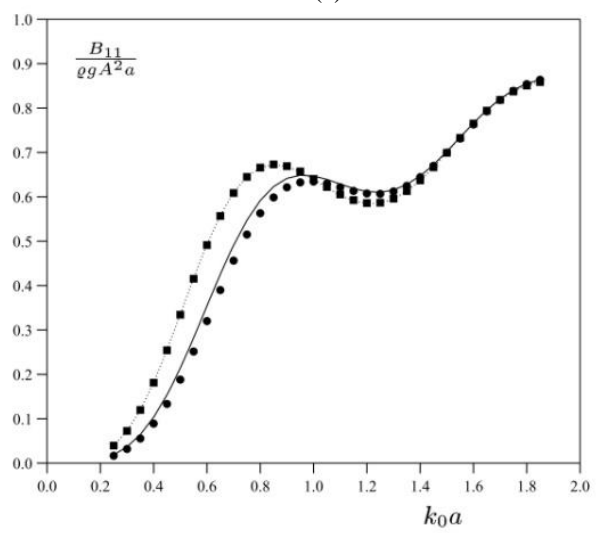

(b)

Fig. 14. Wave drift damping coefficient $B_{11}$ for a fixed truncated cylinder. The cylinder radius is $a$, water depth is $H=5 a$ and wave incidences are $\beta=0$ (a) and $\beta=\pi / 4$. Dotted line: semi- analytic for $D=4 a$; full line: semi-analytic for $D=a$; squres : formula (46) for $D=4 a$; circles: formula (46) for $D=$ $a$.

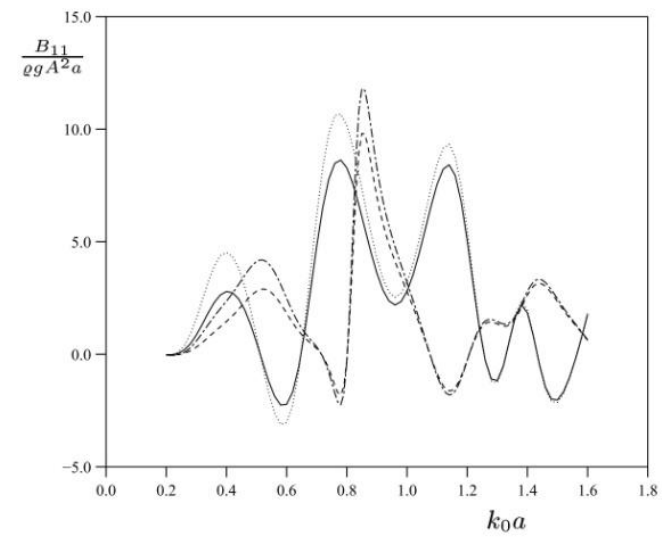

Fig. 15. Wave drift damping coefficient $B_{11}$ for an array of four bottom mounted cylinders of the radius $a$ placed at the corners of the square of side length equal to $7 a$. Dotted line: $\beta$ $=0 ., H=1$; full line: $\beta=0 ., H=\infty$; dot-dashed line: $\beta=\pi / 6$, $H=1 . ;$ dashed line: $\beta=\pi / 6, H=\infty$.

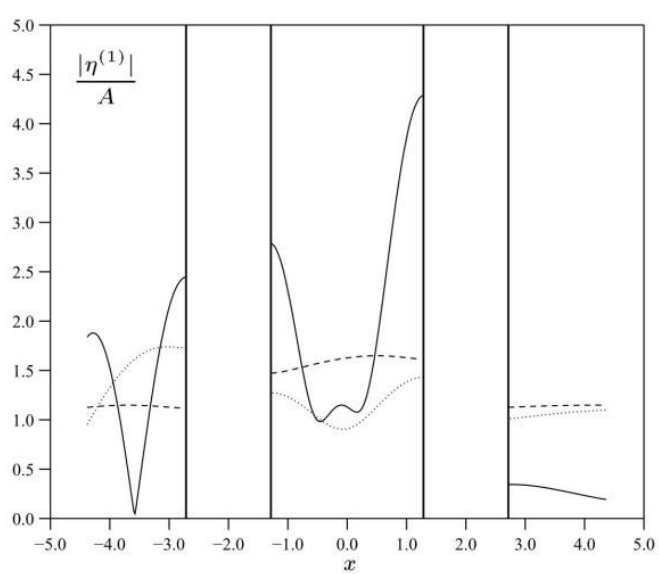

(a)

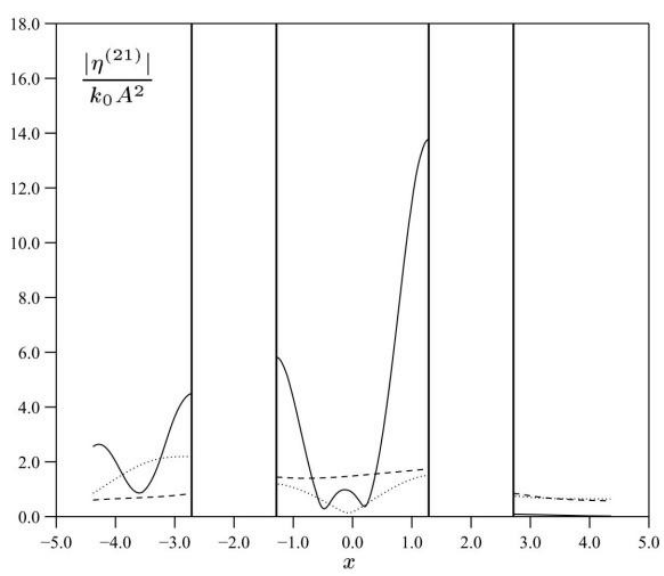

(b)

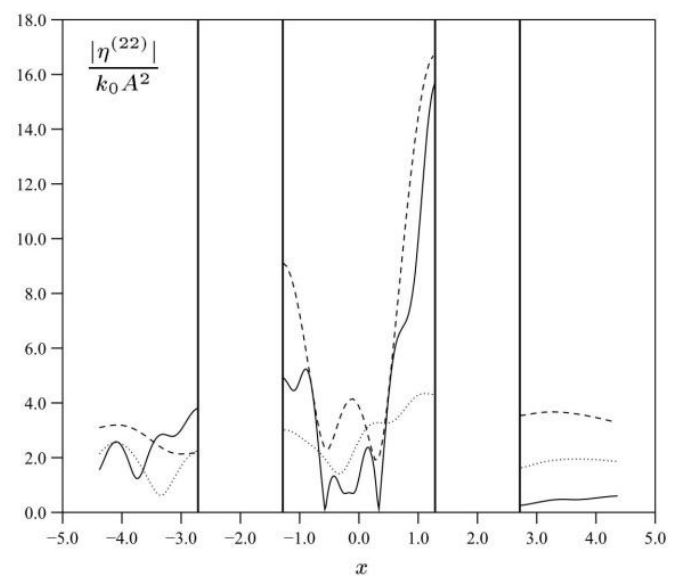

(c) 


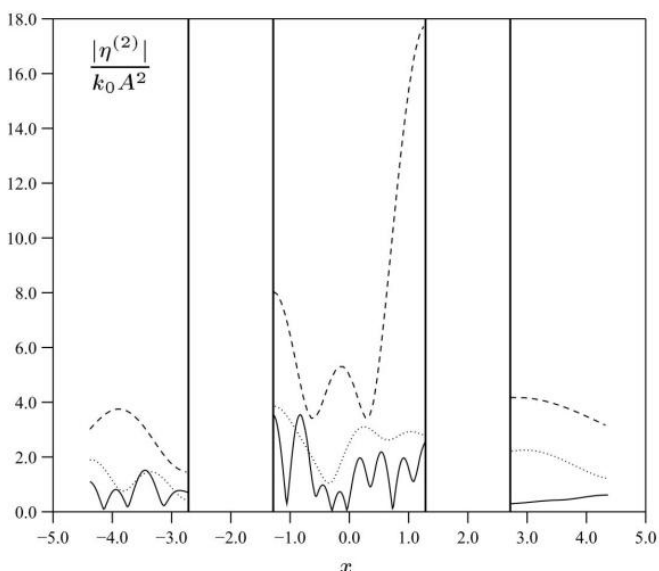

(d)

Fig .16. Components of non-dimensional first and second order wave elevation amplitude along $x=y$ for an array of four bottom mounted cylinders of radius a placed at the corners of the square of side length $4 a$. Wave incidence is $\beta=$ $\pi / 4$. Solid line $k_{0} a=1.66$; dashed line: $k_{0} a=0.468$; dotted line: $k_{0} a=0.754$.

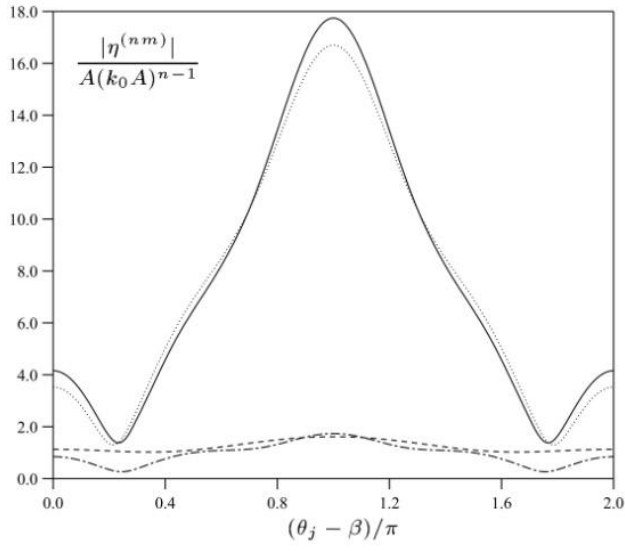

(a)

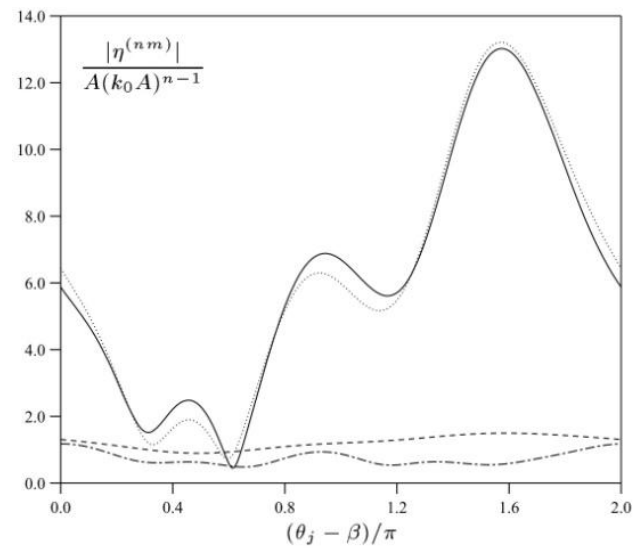

(b)

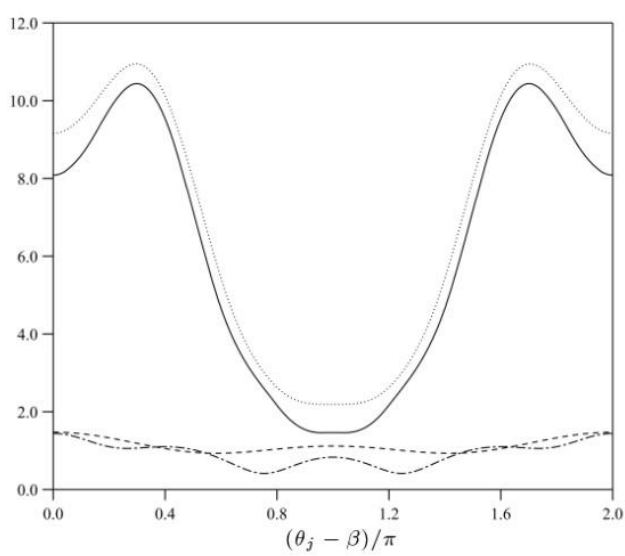

(c)

Fig. 17. Components of non-dimensional first and second order wave run-up amplitude around cylinders for an array of four bottom mounted cylinders of radius a placed at the corners of the square of side length $4 a$. Wave incidence is $\beta=$ $\pi / 4$ and $k_{0} a=1.66$. Dashed line: $\left|\eta^{(1)}\right| / A$; dot-dashed line: $\left|\eta^{(21)}\right|$ $k_{0} A^{2}$; dotted line: $\left|\eta^{(22)}\right| / k_{0} A^{2}$; full line: $\left|\eta^{(2)}\right| / k_{0} A^{2}$. 


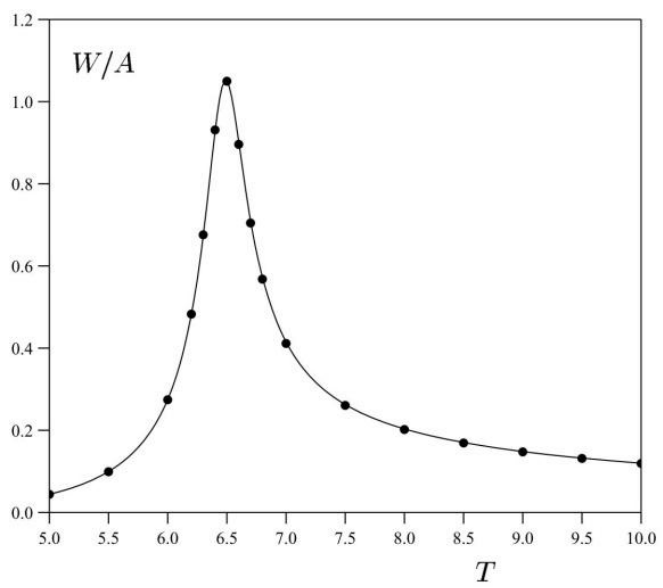

(a)

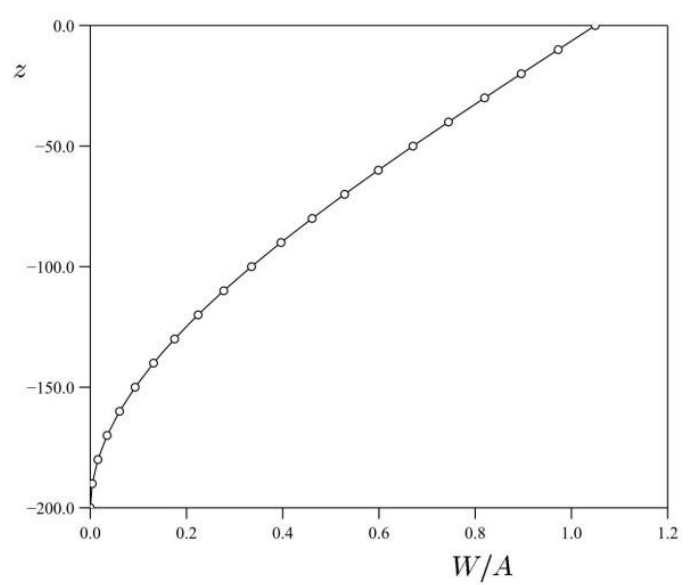

(b)

Fig. 18. Amplitude of the displacement at the top of the column for different wave periods (a) and elastic deformation of the column at $T=6.5 \mathrm{~s}$. The circles on the figure (a) are the results from Newman (1994).

\section{Conclusion}

The methods to treat various kinds of hydrodynamic problems for different cylinder configurations are presented here. Higher order diffraction at zero forward speed, diffraction-radiation at small forward speed and hydroelasticity were considered. The boundary value problems emerging from the different cases are very similar and all of them can be treated by the same methods. the efficiency of these methods is demonstrated by various examples. The analytical methods have their place in the marine hydrodynamics because of their relative simplicity. Due to their high precision, only this kind of method allows the highly nonlinear developments with sufficient confidence. These results are essen- tial for validation of more general numerical codes, which become more and more ambitious due to the rapid computer developments.

\section{References}

[1] Abramowitz M. \& Stegun I., Handbook of mathematical functions, Dover, (1970).

[2] Aranha J.A.P., Second order horizontal steady forces and moment on a floating body with small forward speed, J. Fluid Mech., Vol. 313, (1996) pp. 39-54.

[3] Chau F.P. \& Eatock Taylor R., Second order wave diffraction by a vertical cylinder, J.Fluid Mech., Vol.240, (1992) pp. 571-599.

[4] Chen X.B., Second order high frequency loads on tension leg platform columns, Institut Francais du Petrole, Rep ort No. 38741, (1991).

[5] Chen X.B. \& Malenica Š., Interaction effects of local steady flow on wave diffraction-radiation at low forward speed, Int. Journal of Offshore and Polar Engg., Vol. 8/2, (1998), pp. 102-109.

[6] Noblesse F. \& Chen X.B., Decomposition of free surface effects into wave and near-field components, Ship Technology Res., Vol. 42/4, (1995).

[7] Clark P.J., Š.Malenica \& B.Molin, An heuristic approach to wave drift damping, Appl. Ocean Res., Vol.15, (1993).

[8] Emmerhoff O.J. \& P.D.Sclavounos, The slow drift motion of arrays of vertical cylinders, $\mathrm{J}$. Fluid Mech., Vol.242, (1992) pp. 31-50.

[9] Faltinsen O.M., Sea loads on Ships and Offshore Structures, Cambridge University Press (1990).

[10]Garrett C.J.R., Waves forces on a circular dock, J. Fluid Mech. Vol. 46, (1971) pp. 129139.

[11]Grue J., Personal communication(1993).

[12]Havelock T.H., Forced surface waves on water, Philosophical Magazine 8, (1929) pp. 304311.

[13]John F., On the motion of floating bodies II, Comm. Pure Appl. Math, Vol.3, (1950) pp. 45101.

[14]Linton C.M., Evans D.V., The interaction of waves with arrays of vertical circular cylinders, J.Fluid Mech., Vol.215, (1990) pp. 549569.

[15]Malenica Š. \& S.Etienne, A propos des méthodes semi-analytiques pour les différents 
problèmes de diffraction-radiation par un cylindre tronqué., Proc. of 5ieme Journées de l'Hydro dynamique, Rouen, France (1995).

[16]Malenica Š., P.J.Clark \& B.Molin, 1994. : Wave and current forces on a vertical cylinder free to surge and sway, Appl. Ocean Res., Vol.17. 1995.

[17]Malenica Š., Molin B., Third harmonic wave diffraction by a vertical cylinder, J.Fluid Mech., Vol. 302, (1995) pp. 203-229.

[18]Malenica Š., Some aspects of water wave diffraction-radiation at small forward speed, Brodogradnja, Vol. 45, (1997) pp.35-43.

[19]Malenica S̆., R.Eatock Taylor \& J.B.Huang, Semi-analytical solution for second order water wave diffraction by an array of vertical cylinders, Submitted to J.Fluid Mechanics (1998).

[20]Malenica Š., Hydroelastic coupling of beam structural model with $3 D$ hydro dynamic model, 2nd Int. Conf. on Hydroelasticity, Fukuoka, Japan (1998).

[21]Matsui T., Yeob L.S. \& Kimitoshi S.,:Hydro dynamic forces on a vertical cylinder incurrent and waves, J. of the Soc. of Naval Arch. of Japan, Vol.170, pp. 277-287 (in Japanese), (1991).

[22]Molin B., Second order hydrodynamics applied to moored structures, Ship Tech. Research, Vol. 41/2 (1994).

[23]Newman J.N., Wave effects on deformable bodies, Applied Ocean Research, Vol. 16, (1994) pp.47-59.

[24]Newman J.N., The second order wave force on a vertical cylinder, J.Fluid Mech. (1996)

[25]Noblesse F. \& X.B.Chen, Decomposition of free surface effects into wave and near-field components, Ship Technology Res., Vol. 42/4 (1995).

[26]Nossen J., J.Grue \& E.Palm, Wave forces on three-dimensional floating bodies with small forward speed, J. Fluid Mech., Vol.227,(1991) pp. 135-160.

[27]Senjanovic I., Finite element method in the analysis of ship structures, University Zagreb, Faculty of Mechanical Engg. and Naval Arch (1986). 